
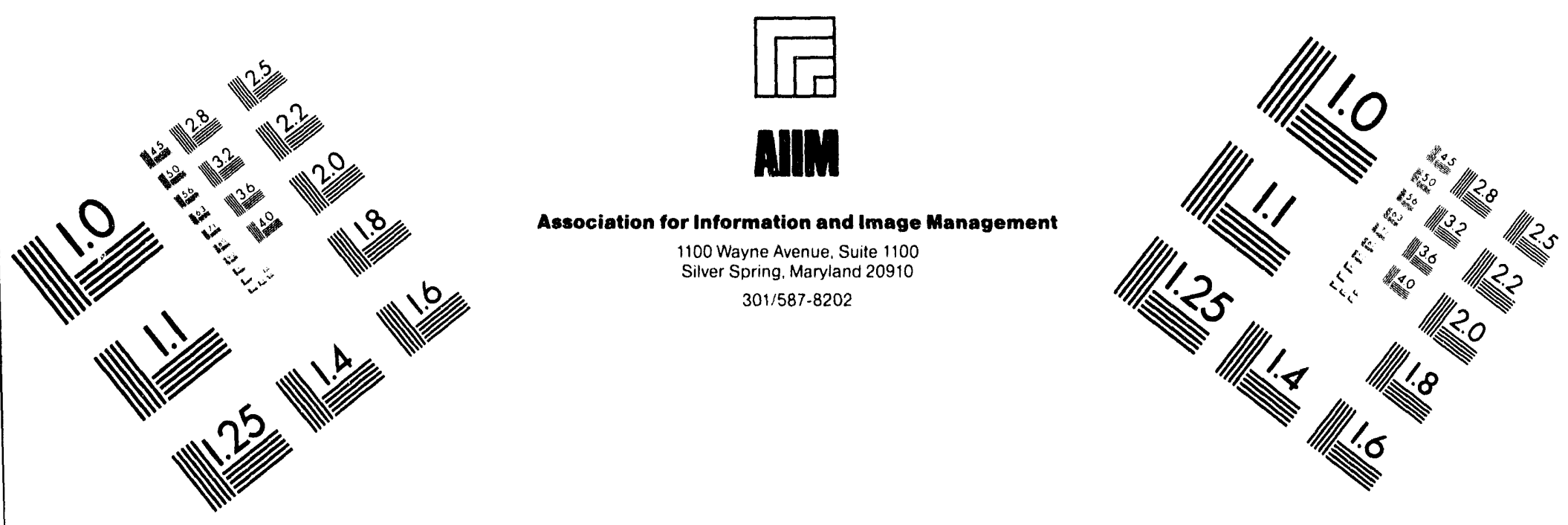

\title{
Centimeter
}

$\begin{array}{llllllllllllllll}1 & 2 & 3 & 4 & 5 & 6 & 7 & 8 & 9 & 10 & 11 & 12 & 13 & 14 & 15 & \mathrm{~mm}\end{array}$

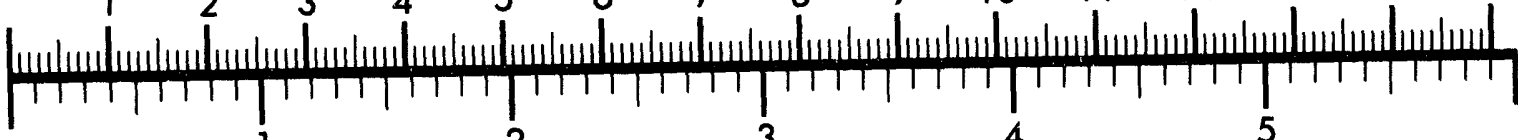
Inches

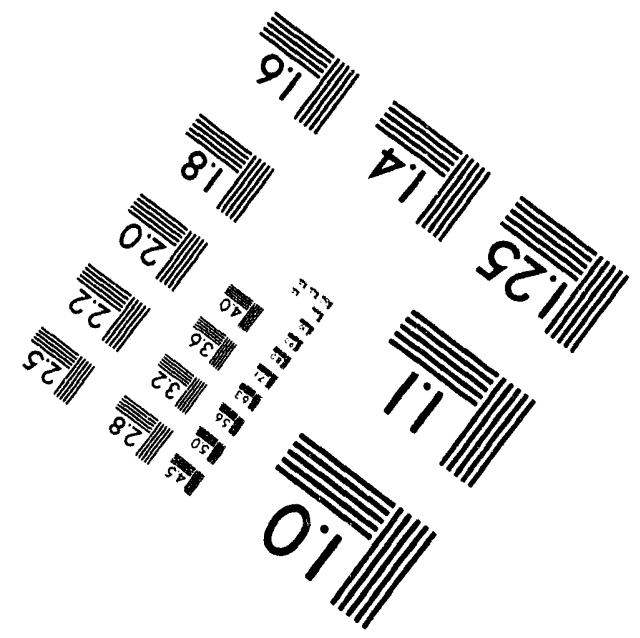

MANUFACTURED TO AIIM STANDARDS BY APPLIED IMAGE. INC.

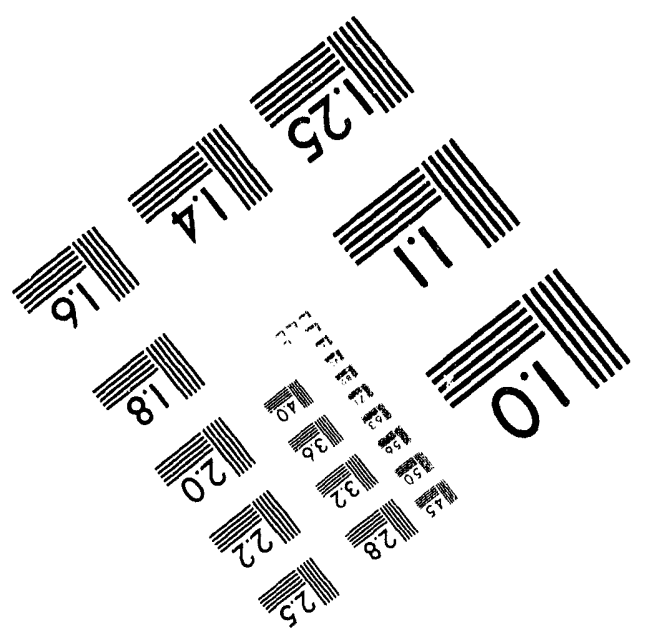



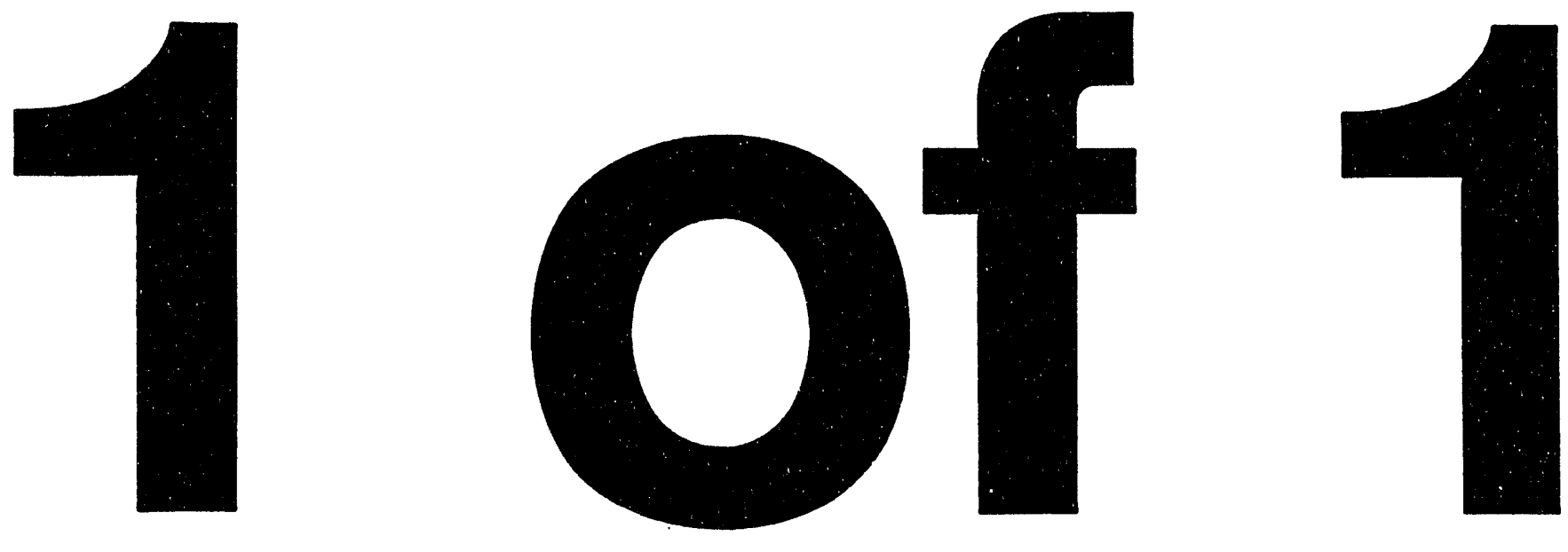
PNL-10073

UC-505

\section{Trigonometric Functions of Nonlinear Quantities}

Dennis W. Wester

August 1994

Prepared for

the U.S. Department of Energy

under Contract DE-AC06-76RLO 1830

Pacific Northwest Laboratory

Richland, Washington 99352 


\begin{abstract}
Trigonometric functions of nonlinear quantities are introduced. Functions of the form. $f\left(x^{a}\right), f\left(x^{y}\right)$, and $f^{n}\left(x^{a}\right)$ are reported, where $f$ is a trigonometric function such as cos, sin, tan, cot, sec, or csc; $x$ is a variable; $a$ is a constant; $y$ is a variable; and $n$ is a constant. Sums, products and quotients of these functions are defined. Trigonometric functions of nonlinear quantities involving constants to variable powers also are mentioned. Possible applications to quantum mechanics, gravity, and a final theory of matter are discussed.
\end{abstract}




\section{Contents}

Abstract $\ldots \ldots \ldots \ldots \ldots \ldots \ldots \ldots \ldots \ldots \ldots \ldots \ldots \ldots \ldots \ldots \ldots \ldots \ldots \ldots$

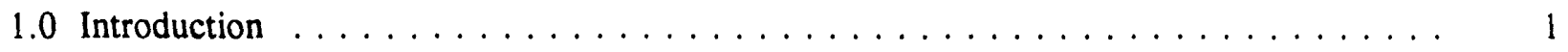

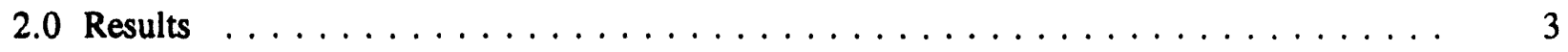

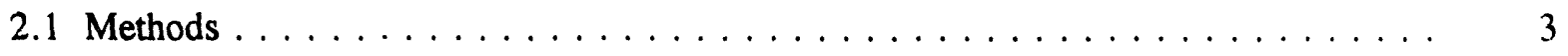

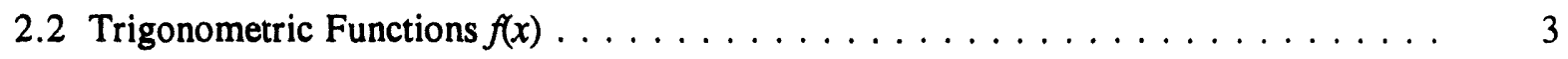

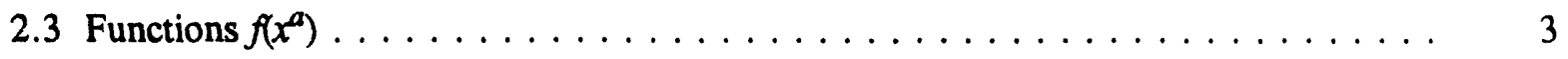

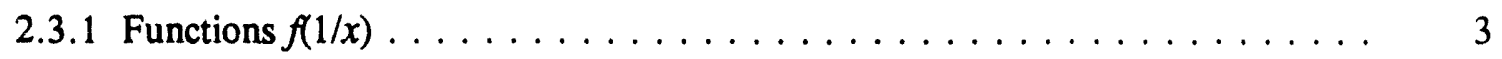

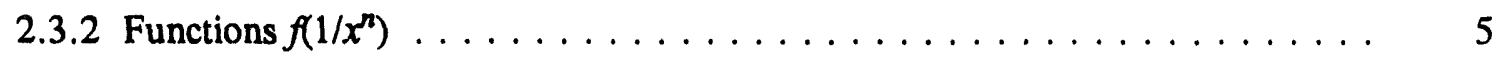

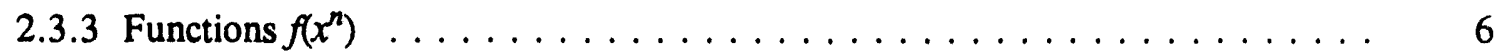

2.3.4 Functions $f\left(x^{y}\right)$, Where $y$ is a Variable . . . . . . . . . . 7

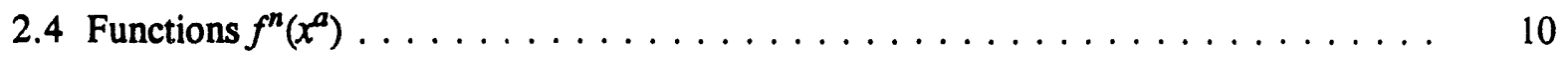

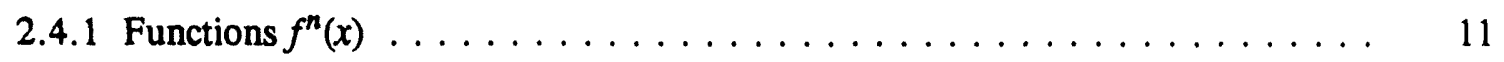

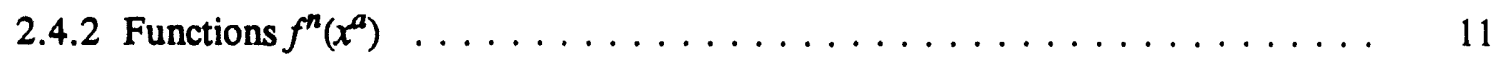

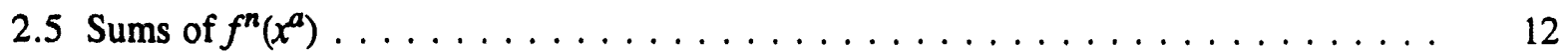

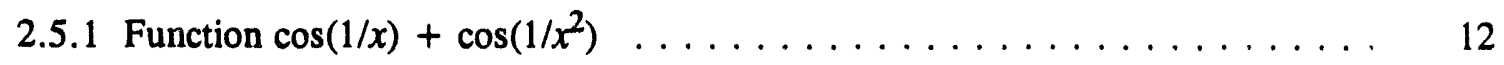

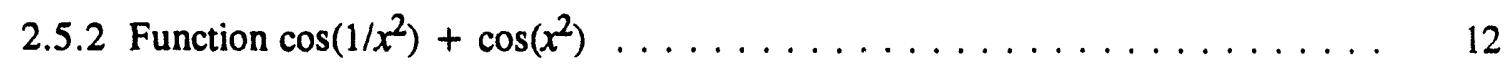

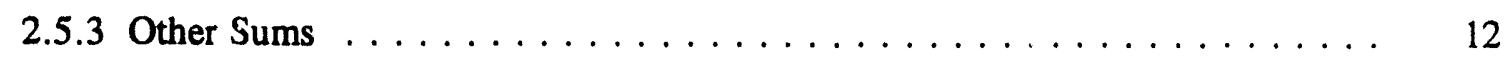

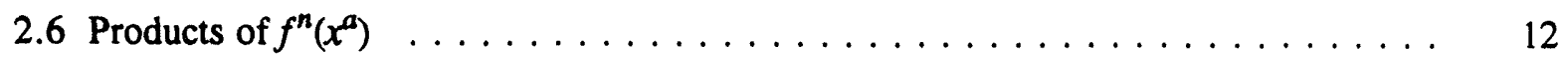

2.6.1 Function $\cos (1 / x) \cdot \cos \left(1 / x^{2}\right) \ldots \ldots \ldots \ldots \ldots \ldots \ldots \ldots$

2.6 .2 Other Products $\ldots \ldots \ldots \ldots \ldots \ldots \ldots \ldots \ldots \ldots$

2.7 Functions $f\left(a^{x}\right), f\left(a^{-x}\right), f\left(y \cdot a^{x}\right)$ and $f\left(y \cdot a^{-x}\right) \ldots \ldots \ldots \ldots$

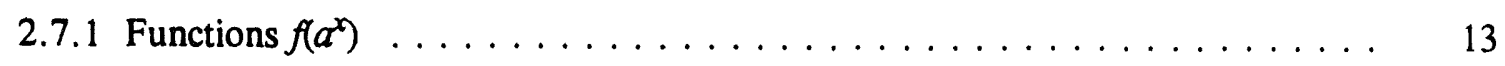




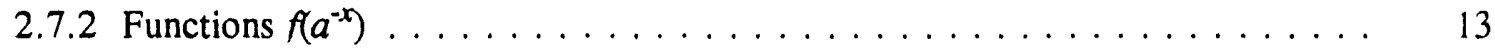

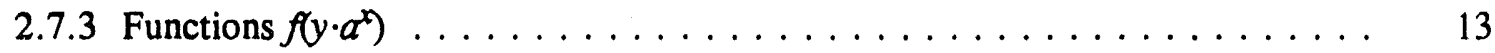

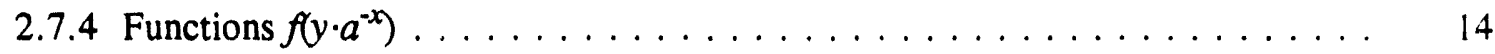

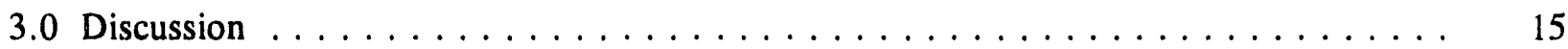

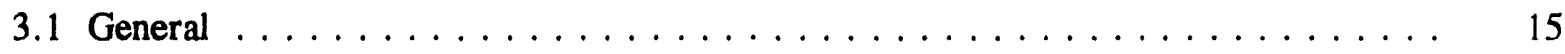

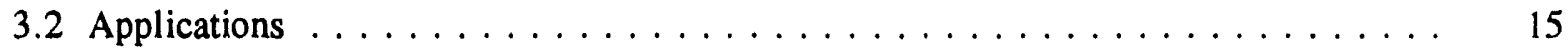

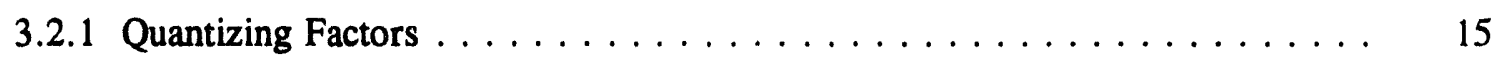

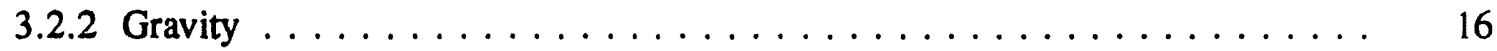

3.2 .3 Final Theory $\ldots \ldots \ldots \ldots \ldots \ldots \ldots \ldots \ldots \ldots \ldots \ldots \ldots \ldots$

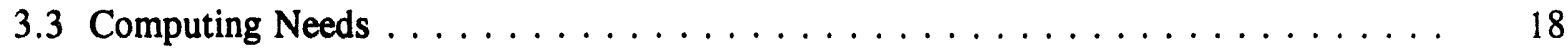

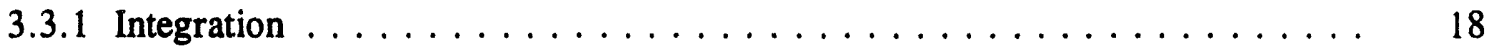

3.3.2 Curve Fitting $\ldots \ldots \ldots \ldots \ldots \ldots \ldots \ldots \ldots \ldots \ldots \ldots$

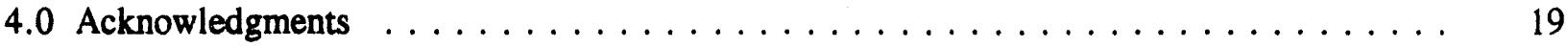




\section{Figures}

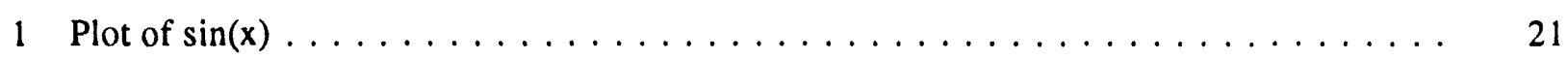

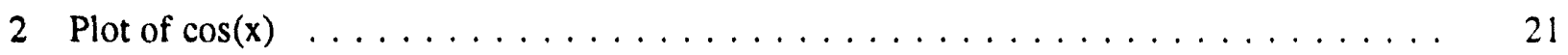

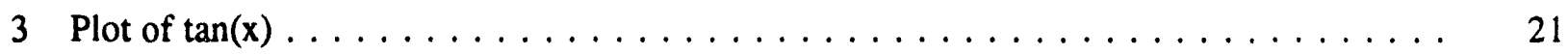

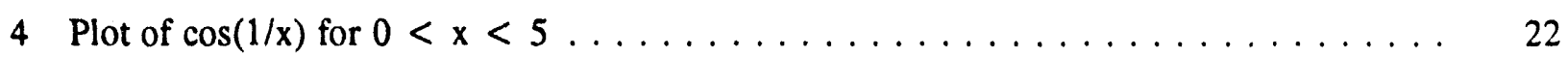

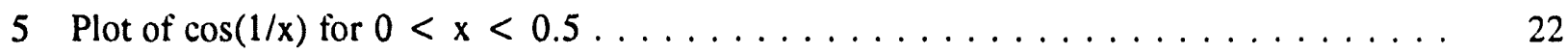

6 Plot of $\cos (1 / \mathrm{x})$ for $0<\mathrm{x}<0.05 \ldots \ldots \ldots \ldots \ldots \ldots$

7 Plot of $\sin (1 / x)$ for $0<x<5 \ldots \ldots \ldots \ldots \ldots \ldots \ldots$

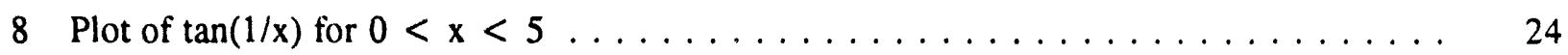

9 Plot of $\cot (1 / \mathrm{x})$ for $0<\mathrm{x}<1 \ldots \ldots \ldots \ldots \ldots \ldots \ldots \ldots$

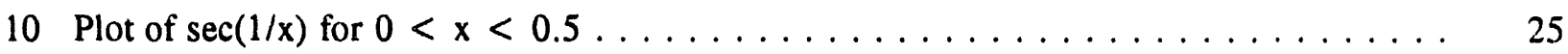

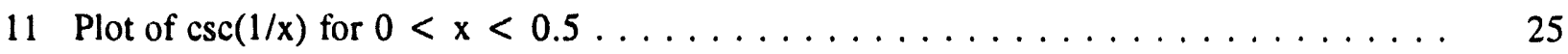

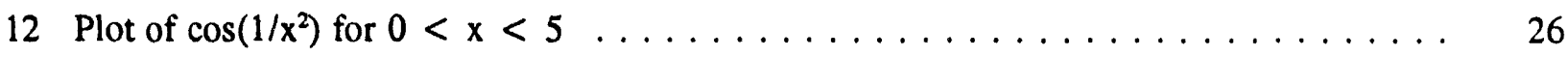

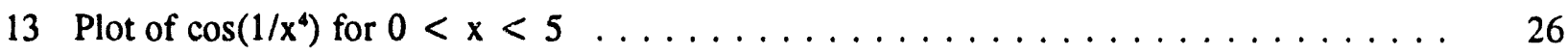

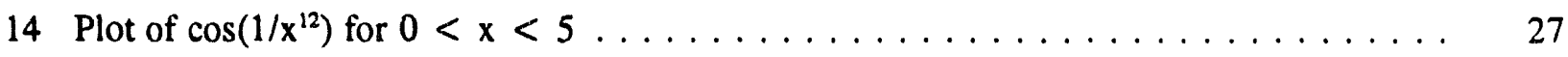

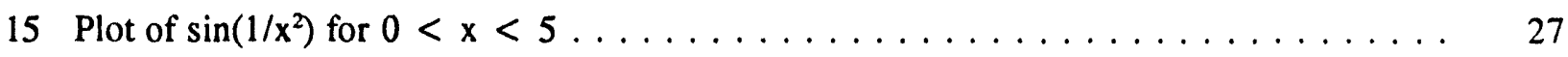

16 Plot of $\tan \left(1 / \mathrm{x}^{4}\right)$ for $0<\mathrm{x}<5 \ldots \ldots \ldots \ldots \ldots \ldots \ldots$

17 Plot of $\cos \left(\mathrm{x}^{2}\right)$ for $0<\mathrm{x}<10 \ldots \ldots \ldots \ldots \ldots \ldots$

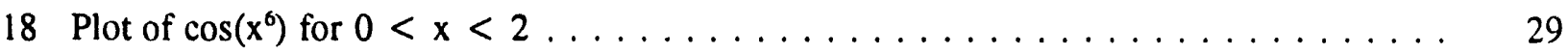

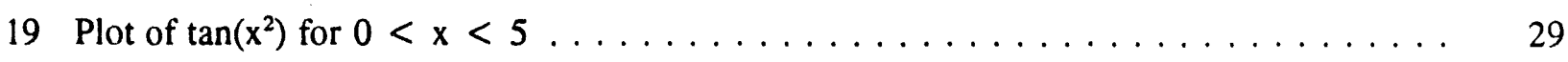

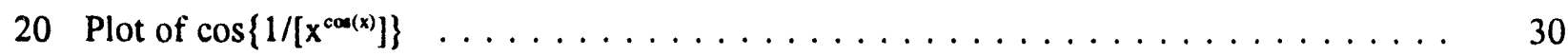

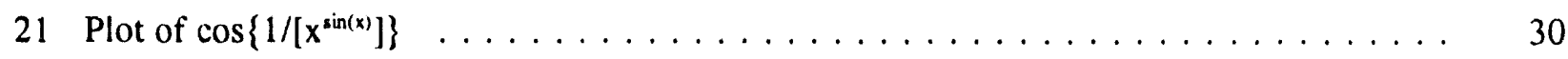

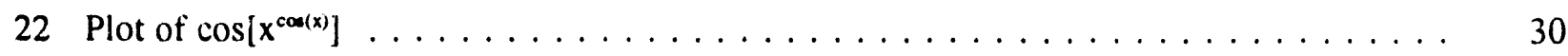




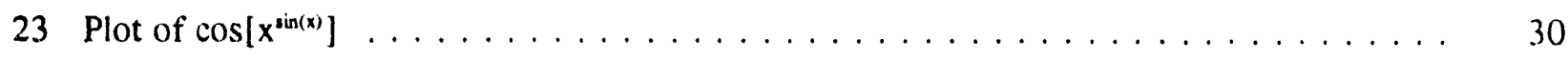

24 Plot of $\cos (1 / x) \cdot \cos (1 / x)$ for $0<x<5 \ldots \ldots \ldots \ldots \ldots$

25 Plot of $\cos (1 / x)+\cos \left(1 / x^{2}\right)$ for $0<x<1 \ldots \ldots \ldots 31$

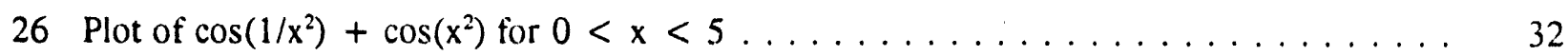

27 Plot of $\cos (1 / x) \cdot \cos \left(1 / x^{2}\right)$ for $0<x<1 \ldots \ldots \ldots \ldots 2$

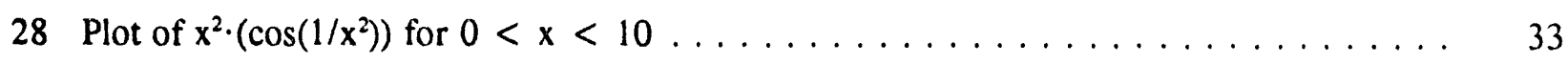

29 Plot of $x^{2} \cdot\left(\cos \left(1 / x^{2}\right)\right)$ for $0<x<1 \ldots \ldots \ldots \ldots$

30 Plot of $x^{2} \cdot\left(\cos \left(1 / x^{2}\right)\right)$ for $0<x<0.1 \ldots \ldots \ldots \ldots \ldots$ 


\subsection{Introduction}

The ability to describe physical phenomena by using mathematical equations has fascinated mankind almost since the beginning of recorded history. The trigonometric functions are some of the earliest that were known. Throughout history, these functions have been applied to some of the most daunting problems. A common thread in all these applications is that the arguments of the trigonometric functions are linear functions of angles in one form or another.

A search for functions with properties that would reliably reproduce such physical phenomena as the shift from particle to wave nature as matter becomes less and less massive and the seemingly discontinuous nature of matter itself revealed that suitable functions had not been reported. Therefore, an attempt was made to invent such functions.

In this report, the concept of the application of trigonometric functions to nonlinear quantities is introduced. This concept expands the applicability of the trigonometric functions to areas that heretofore have been unexplored. In the discussion, the application of these new functions to the problems mentioned abcve are noted. 


\subsection{Results}

\subsection{Methods}

Calculations and plots in this article were made using the software program SigmaPlot (Jandel Scientific, Version 1.02) on a Zeos 486 personal computer, or using a Hewlett Packard 48SX Scientific Expandable pocket calculator. In all instances, the argument of the trigonometric function was expressed in radians. In several instances the plotting capabilities of the program were inadequate to portray the rapid oscillations of the functions as limits of $x$ were approached. In general, these plots contain peaks or valleys that do not reach the limiting value on the ordinate or are altogether missing peaks and valleys that should appear at regular intervals. In each instance, the overall shape of the plot is sufficient to demonstrate the trend in the spacing between the peaks and valleys.

\subsection{Trigonometric Functions $f(x)$}

For sake of completeness, the common trigonometric functions of linear quantities are first treated. Thus, the well-known functions $\sin (x), \cos (x)$, and $\tan (x)$ are plotted in Figures 1 through 3 . As expected, it can be seen from Figure 1 that the function $\sin (x)$ has a value of 0 at $x=\ldots-3 \pi,-2 \pi$, $-\pi, 0, \pi, 2 \pi, 3 \pi \ldots$; of 1 at $x=\ldots-3 \pi / 2, \pi / 2,5 \pi / 2 \ldots$; and of -1 at $x=\ldots-5 \pi / 2,-\pi / 2,3 \pi / 2 \ldots$ This result is consistent with the antisymmetric nature of $\sin (x)$ around the origin. On the other hand, Figure 2 shows that the function $\cos (x)$ has a value of 0 at $x=\ldots-5 \pi / 2,-3 \pi / 2,-\pi / 2, \pi / 2,3 \pi / 2$, $5 \pi / 2 \ldots$; of -1 at $x=\ldots-3 \pi,-\pi, \pi, 3 \pi \ldots$; and of 1 at $x=\ldots-2 \pi, 0,2 \pi \ldots$ This result is consistent with the symmetric nature of $\cos (x)$ around the origin. Finally, Figure 3 shows the plot of $\tan (x)$, which has a value of 0 at $x=\ldots-3 \pi,-2 \pi,-\pi, 0, \pi, 2 \pi, 3 \pi \ldots$ and is undefined $(\infty)$ at $x=\ldots-5 \pi / 2,-3 \pi / 2,-\pi / 2, \pi / 2,3 \pi / 2,5 \pi / 2 \ldots$, consistent with the expected behavior of this function. It is important to note that, in all instances, the trigonometric functions are operating on linear quantities; that is, the number of radians depends linearly on the quantity $x$. The functions $\csc (x)$, $\sec (x)$, and $\cot (x)$ are easily derived by taking the inverse of $\sin (x), \cos (x)$, and $\tan (x)$, respectively.

\subsection{Functions $f\left(x^{a}\right)$}

For these functions, where $f$ is one of the trigonometric functions, the quantity $a$ can be any real number or a variable. Thus, $a$ can be a negative integer such as $-1,-2,-3 \ldots$; a positive integer such as $1,2,3 \ldots$; a fraction such as $2 / 3,3 / 4,3 / 10$, etc.; or any negative or positive decimal quantity. In addition, $a$ can be a variable represented by, for example, a function of $x$.

\subsubsection{Functions $f(1 / x)$}

These functions represent instances of $f\left(x^{a}\right)$, where $a=-1$. 


\subsubsection{Function $\cos (1 / x)$}

To illustrate the behavior of functions such as $f\left(x^{a}\right)$, where $a=-1, \cos (1 / x)$ is plotted in Figures 4 through 6 for several ranges of $x$. In Figure 4, the range is $x=0$ to 5 (radians). Note that the function has a value of 0 at $x=2 / \pi, 2 / 3 \pi, 2 / 5 \pi \ldots$; of -1 at $x=1 / \pi, 1 / 3 \pi, 1 / 5 \pi \ldots$; and of 1 at $x=1 / 2 \pi, 1 / 4 \pi \ldots$. Other ranges of $x$ are shown in Figures 5 and 6 , respectively, so that the details of the function as $x \rightarrow 0$ can be seen more clearly. Again, note that $\cos (1 / x)$ in Figure 5 has a value of 0 at $x=2 / 3 \pi, 2 / 5 \pi, 2 / 7 \pi, \ldots$ (the quantity $x=2 / \pi$ is off the scale); of -1 at $x=1 / \pi, 1 / 3 \pi, 1 / 5 \pi \ldots$; and of 1 at $x=1 / 2 \pi, 1 / 4 \pi, 1 / 6 \pi \ldots$. In other words, the function $\cos (1 / x)$ has a value of 0 at $x=2 /(2 m+1) \pi$; of -1 at $x=1 /(2 m+1) \pi$; and of 1 at $x=1 / 2 m \pi$, where $m$ is a positive integer or 0 . The function $\cos (1 / x)$ thus oscillates more and more rapidly between the values of 1 and -1 as $x \rightarrow 0$. This is clearly illustrated in Figure 6, where the function $\cos (1 / x)$ begins to oscillate rapidly as $x \rightarrow 0$ owing to the rapid oscillation between the quantities $x=2 /(2 m+1) \pi, 1 /(2 m+1) \pi$, and $1 / 2 m \pi$. Note in Figure 4 that the function $\cos (1 / x) \rightarrow 1$ as $x \rightarrow \infty(m=0$ for $x=1 / 2 m \pi)$.

\subsubsection{Function $\sin (1 / x)$}

Figure 7 shows that $\sin (1 / x)$ has a value of 0 at $x=\infty, 1 / \pi, 1 / 2 \pi, 1 / 3 \pi \ldots(1 / m \pi)$; of 1 at $x=2 / \pi$, $2 / 5 \pi, 2 / 9 \pi \ldots 2 /(4 m+1) \pi$; and of -1 at $x=2 / 3 \pi, 2 / 7 \pi, 2 / 11 \pi \ldots 2 /(4 m+3) \pi$. Like for the function $\cos (1 / x), \sin (1 / x)$ begins to oscillate rapidly as $x \rightarrow 0$ owing to the rapid oscillation between the quantities $1 / m \pi, 2 /(4 m+1) \pi$, and $2 /(4 m+3) \pi$. Note that the function $\sin (1 / x) \rightarrow 0$ as $x \rightarrow \infty$.

\subsubsection{Function $\tan (1 / x)$}

As shown in Figure 8, the function $\tan (1 / x)$ exhibits the now-familiar rapid oscillation as $x \rightarrow 0$. Thus, $\tan (1 / x)$ has a value of 0 at $x=1 / \pi, 1 / 2 \pi, 1 / 3 \pi \ldots(1 / m \pi)$ and is undefined at $x=2 / \pi, 2 / 3 \pi$, $2 / 5 \pi \ldots .2 /(2 m+1) \pi$. Note that $\tan (1 / x) \rightarrow 0$ as $x \rightarrow \infty$.

\subsubsection{Function $\cot (1 / x)$}

Figure 9 shows a plot of the inverse of $\tan (1 / x), \cot (1 / x)$. As expected, $\cot (1 / x)$ has a value of 0 at $x=2 / \pi, 2 / 3 \pi, 2 / 5 \pi \ldots 2 /(2 m+1) \pi$ and is undefined at $1 / \pi, 1 / 2 \pi, 1 / 3 \pi \ldots(1 / m \pi)$; that is, $\cot \left({ }^{\prime} / x\right)$ has a value of 0 at those points where $\tan (1 / x)$ is undefined and vice versa. Also note, although not clearly shown in Figure 9, that $\cot (1 / x)$ becomes undefined as $x \rightarrow \infty$.

\subsubsection{Function $\sec (1 / x)$}

Figure 10 shows a plot of the inverse of $\cos (1 / x), \sec (1 / x)$. In this instance, $\sec (1 / x)$ is undefined at those points where $\cos (1 / x)$ has a value of 0 ; that is, $2 / \pi$ (off the scale), $2 / 3 \pi, 2 / 5 \pi \ldots 2 /(2 m+1) \pi$. The function $\sec (1 / x)$ has a value of -1 at those points where $\cos (1 / x)$ has a value of -1 , that is, $1 / \pi$, $1 / 3 \pi, 1 / 5 \pi \ldots 1 /(2 m+1) \pi$, and a value of 1 at those points where $\cos (1 / x)$ has a value of 1 , that is, $1 / 2 \pi, 1 / 4 \pi, \ldots(1 / 2 m \pi)$. Although not clearly shown in Figure 10, as $x \rightarrow \infty, \sec (1 / x) \rightarrow 1$.

\subsubsection{Function $\csc (1 / x)$}

Figure 11 shows a plot of $\csc (1 / x)$. Because $\csc (1 / x)$ is the inverse of $\sin (1 / x)$, the function is undefined at those points where $\sin (1 / x)$ has a value of 0 , that is, $1 / \pi, 1 / 2 \pi, 1 / 3 \pi \ldots(1 / m \pi)$; has a value 
of -1 at those points where $\sin (1 / x)$ has a value of -1 , that is, $2 / 3 \pi, 2 / 7 \pi, 2 / 11 \pi \ldots 2 /(4 m+3) \pi$; and has a value of 1 at those points where $\sin (1 / x)$ has a value of 1 , that is, $2 / \pi$ (off the scale), $2 / 5 \pi$, $2 / 9 \pi \ldots 2 /(4 m+1) \pi$. Although not clearly shown in Figure $11, \csc (1 / x)$ becomes undefined as $x \rightarrow \infty$.

\subsubsection{Functions $f\left(1 / x^{n}\right)$}

These functions represent the general functions $f\left(x^{a}\right)$, where $a$ is a negative integer.

\subsubsection{Function $\cos \left(1 / x^{n}\right)$}

The function $\cos (1 / x)$ represents the trivial case of a much broader function, $\cos \left(1 / x^{n}\right)$, where $n=1$. The effect of raising the quantity $1 / x^{n}$ to a positive integral power is clearly seen in the series of plots shown in Figures 12 through 14. A comparison of these figures and Figure 4 demonstrates that the function $\cos \left(1 / x^{n}\right)$ begins to oscillate more rapidly at larger values of $x$ as $n \rightarrow \infty$. Thus, the largest value of $x$ at which the function $\cos (1 / x)$ reaches a value of -1 is $x=1 / \pi$. However, owing to the exponent of 2 , the largest value of $x$ at which $\cos \left(1 / x^{2}\right)$ reaches a value of -1 is $x=(1 / \pi)^{1 / 2}$, as shown in Figure 12. Similarly, the largest values of $x$ at which the functions $\cos \left(1 / x^{4}\right)$ and $\cos \left(1 / x^{12}\right)$ reach a value of -1 are $x=(1 / \pi)^{1 / 4}$ and $(1 / \pi)^{1 / 12}$, respectively. This behavior can be seen by comparing the plots shown in Figures 13 and 14. In Figure 14, the function $\cos \left(1 / x^{12}\right)$ begins to oscillate so rapidly as $x \rightarrow 0$ that the capabilities of the plotting program are exceeded and a value of 0 is plotted.

It should be mentioned that in each instance the largest value of $x$ at which the function $\cos \left(1 / x^{n}\right)$ reaches a value of 0 is $1 / x^{n}=2 / \pi$. Moreover, as $n \rightarrow \infty, 1 / x^{n} \rightarrow 2 / \pi$ at values of $x$ approaching 1 , because $1 / x=(2 / \pi)^{1 / n}$ and $(2 / \pi)^{1 / n} \rightarrow 1$ as $n \rightarrow \infty$.

Similar arguments can be made to explain the fact that $\cos \left(1 / x^{n}\right)$ reaches the asymptote of 1 at lower values of $x$ as $n \rightarrow \infty$.

\subsubsection{Other Functions $f\left(x^{\mathfrak{n}}\right)$, Where $a$ is a Negative Integer}

By analogy with the functions $f(1 / x)$, where $a=-1$ (Sections 2.3.1.1 through 6 ), and $\cos \left(1 / x^{n}\right)$, where $n$ is a negative integer (Section 2.3.2.1), it can be shown that each of the functions $\sin \left(1 / x^{n}\right)$, $\tan \left(1 / x^{n}\right), \cot \left(1 / x^{n}\right), \sec \left(1 / x^{n}\right)$, and $\csc \left(1 / x^{n}\right)$ begin to oscillate more rapidly as $n \rightarrow \infty$. This is shown for $\sin \left(1 / x^{2}\right)$ in Figure 15 and for $\tan \left(1 / x^{4}\right)$ in Figure 16.

Function $\sin \left(1 / x^{n}\right)$. A comparison of Figure 15 with Figure 7 shows that the function $\sin \left(1 / x^{n}\right)$ will reach values of 1 at greater values of $x$ and 0 at lower values of $x$ as $n \rightarrow \infty$. For $\sin \left(1 / x^{n}\right)$, the function has a value of 1 for $1 / x^{n}=2 / \pi$. Thus, the value of the function approaches 1 at values of $x$ approaching 1 as $n \rightarrow \infty$, because $1 / x=(2 / \pi)^{1 / n}$ and $(2 / \pi)^{1 / n} \rightarrow 1$ as $n \rightarrow \infty$. The function has a value of 0 for $1 / x^{n}=1 / \pi$. Thus, the value of the function approaches 0 at values of $x$ approaching 1 as $n \rightarrow \infty$, because $1 / x=(1 / \pi)^{1 / n}$ and $(1 / \pi)^{1 / n} \rightarrow 1$ as $n \rightarrow \infty$.

Function $\tan \left(1 / x^{n}\right)$. For the function $\tan \left(1 / x^{n}\right)$, the plots of $\tan (1 / x)$ (Figure 8) and $\tan \left(1 / x^{4}\right)$ (Figure 16) are compared. As expected, the function $\tan \left(1 / x^{n}\right)$ begins to oscillate more rapidly as $n \rightarrow \infty$. In this instance, the largest value of $x$ at which $\tan \left(1 / x^{n}\right)$ first becomes undefined is $1 / x^{n}=2 / \pi$. Thus, the largest value of $x$ at which $\tan \left(1 / x^{n}\right)$ becomes undefined approaches 1 as $n \rightarrow \infty$, because $1 / x=(2 i \pi)^{1 / n}$ and $(2 / \pi)^{1 / n} \rightarrow 1$ as $n \rightarrow \infty$. 
Similar arguments can be made to explain the fact that $\tan \left(1 / x^{n}\right)$ reaches the asymptote of 0 at lower values of $x$ as $n \rightarrow \infty$.

\subsubsection{Functions $f\left(x^{a}\right)$, Where $a<-1$}

These functions include those for which $a$ is a negative integer and those for which $a$ is a fraction or decimal number less than -1 . As a rule, the plots of these functions represent instances that are intermediate between those already mentioned in, for example, Sections 2.3.1 and 2.3.2. Therefore, these functions will not be presented as plots. However, it is important to note that these functions are possible, because applications of this theory may require that the exponent be fitted to the best value, which may not always be an integer.

\subsubsection{Functions $f\left(x^{a}\right)$, Where $-1<a<0$}

These are represented by such functions as $\cos \left(1 / x^{0.9}\right), \cos \left(1 / x^{0.5}\right), \cos \left(1 / x^{0.1}\right), \tan \left(1 / x^{0.9}\right)$, $\tan \left(1 / x^{0.5}\right), \tan \left(1 / x^{0.1}\right)$, etc. In general, those functions with exponents close to 1 resemble the functions $f(1 / x)$. As the exponent approaches 0 , the functions become more elongated along the $x$ axis at higher values of $x$, and more compressed along the $x$ axis at lower values of $x$. Thus, $\cos \left(1 / x^{0.1}\right)$ reaches a value of $\sim 0.922$ only at $x=10^{4}$ whereas $\cos \left(1 / x^{0.9}\right)$ is already $\sim 0.992$ at $\sim 5.23$. Conversely, at small values of $x$ (less than 0.2$)$, where $\cos \left(1 / x^{0.9}\right)$ exhibits several oscillations, $\cos \left(1 / x^{0.1}\right)$ exhibits only a smooth curve very slowly approaching the asymptote of 1 in this range. The function $\cos \left(1 / x^{0.1}\right)$ crosses the abscissa for the last time as $x$ increases at $\sim 0.011$, and oscillations become significant only for exceedingly small values of $x$, for example, $10^{-15}$.

\subsubsection{Functions $f\left(x^{n}\right)$}

These functions represent the general functions $f\left(x^{a}\right)$, where $a$ is a positive number. For the trivial case $a=1$, the common trigonometric functions are obtained. These were discussed in Section 2.2. For instances where $n$ is an integer greater than 1, nonlinear plots of the trigonometric functions are obtained. Several of these are discussed below.

\subsubsection{Function $\cos \left(x^{n}\right)$}

The function $\cos \left(x^{n}\right)$ will be used as one example to illustrate the behavior of the trigonometric functions of $x^{n}$ where $n$ is a positive integer greater than 1. Figure 17 shows a plot of the function $\cos \left(x^{2}\right)$. It can easily be seen that the function begins to oscillate more and more rapidly as $x \rightarrow \infty$. Thus, the function $\cos \left(x^{2}\right)$ has a value of 0 at $x=(\pi / 2)^{1 / 2},(3 \pi / 2)^{1 / 2},(5 \pi / 2)^{1 / 2} \ldots[(2 m+1) \pi / 2]^{1 / 2}$; of -1 at $x=(\pi)^{1 / 2},(3 \pi)^{1 / 2},(5 \pi)^{1 / 2} \ldots[(2 m+1) \pi]^{1 / 2}$; and of 1 at $x=0,(2 \pi)^{1 / 2},(4 \pi)^{1 / 2} \ldots(2 m \pi)^{1 / 2}$. This behavior is just the opposite of that seen for the functions $\cos \left(1 / x^{n}\right)$, where the oscillations become more and more rapid as $x \rightarrow 0$.

For higher powers of $x^{n}$, the function $\cos \left(x^{6}\right)$ will be used to illustrate the behavior as $n \rightarrow \infty$. Figure 18 shows a plot of the function $\cos \left(x^{6}\right)$. It can be seen that the function oscillates more rapidly at lower values of $x$ than $\cos \left(x^{2}\right)$. Thus, $\cos \left(x^{6}\right)$ has a value of 0 at $x=(\pi / 2)^{1 / 6},(3 \pi / 2)^{1 / 6}$, $(5 \pi / 2)^{1 / 6} \ldots[(2 m+1) \pi / 2]^{1 / 6} ;$ of -1 at $x=(\pi)^{1 / 6},(3 \pi)^{1 / 6},(5 \pi)^{1 / 6},[(2 m+1) \pi]^{1 / 6}$; and of 1 at $x=0$, $(2 \pi)^{1 / 6},(4 \pi)^{1 / 6} \ldots(2 m \pi)^{1 / 6}$. Like for the inverse power reiationships of Section 2.3 .2 , the smallest value of $x$ at which the function approaches 0 approaches 1 as $n \rightarrow \infty$, because $(\pi / 2)^{1 / n} \rightarrow 1$ as $n \rightarrow \infty$. 


\subsubsection{Function $\tan \left(x^{n}\right)$}

Another example illustrating the behavior of functions of the form $f\left(x^{a}\right)$ is the function $\tan \left(x^{2}\right)$. As seen in Figure 19, this function begins to oscillate more and more rapidly as $x \rightarrow \infty$. The function $\tan \left(x^{2}\right)$ has a value of 0 at $x=0,(\pi)^{1 / 2},(2 \pi)^{1 / 2} \ldots(m \pi)^{1 / 2}$ and is undefined at $x=(\pi / 2)^{1 / 2}$, $(3 \pi / 2)^{1 / 2} \ldots[(2 m+1) \pi / 2]^{1 / 2}$.

Although not illustrated, the lowest value of $x$ at which the function $\tan \left(x^{n}\right)$ will be undefined approaches 1 as $n \rightarrow \infty$, because $(\pi / 2)^{1 / n} \rightarrow 1$ as $n \rightarrow \infty$.

\subsubsection{Other Functions $f\left(x^{n}\right)$}

In analogy with the functions $f\left(1 / x^{n}\right)$, it can easily be demonstrated that $\sin \left(x^{n}\right), \cot \left(x^{n}\right), \sec \left(x^{n}\right)$, and $\csc \left(x^{n}\right)$ exhibit behavior similar to that of $\cos \left(x^{n}\right)$ and $\tan \left(x^{n}\right)$. Thus, these functions oscillate more and more rapidly as $x \rightarrow \infty$ for a given value of $n$ and the oscillations become more and more rapid at lower values of $x$ as $n \rightarrow \infty$.

\subsubsection{Functions $f\left(x^{m}\right)$, Where $a>1$}

These functions include those for which $a$ is a positive integer and those for which $a$ is a fraction or decimal number greater than 1 . As a rule, the plots of these functions represent instances that are intermediate between those already mentioned in, for example, Sections 2.3.3.1 through 2.3.3.j Therefore, these functions will not be presented as plots. However, it is important to note that tivese functions are possible, because applications of this theory may require that the exponent be fitted to the best value, which may not always be an integer.

\subsubsection{Functions $f\left(x^{a}\right)$, Where $0<a<1$}

These are represented by such functions as $\cos \left(x^{0.9}\right), \cos \left(x^{0.5}\right), \cos \left(x^{0.1}\right), \tan \left(x^{0.9}\right), \tan \left(x^{0.5}\right)$, $\tan \left(x^{0.1}\right)$, etc. In general, those functions with exponents close to 1 resemble the functions $f(x)$. Thus, $\cos \left(x^{0.9}\right)$ very closely resembles $\cos (x)$, crossing the abscissa at values of $x$ increasingly greater than

$(2 m+1) \pi$ as $x$ increases. As the exponent approaches 0 , the functions become more elongated along the $x$ axis. Thus, $\cos \left(x^{0.1}\right)$ crosses the abscissa for the first time as $x$ increases at $(\pi / 2)^{10}(x=\sim 91)$.

\subsubsection{Functions $f\left(x^{y}\right)$, Where $y$ is a Variable}

In addition to the aforementioned functions in which the exponent is a constant, functions also can be constructed in which the exponent itself is a variable. For sake of clarity, the examples that follow will use exponents that are variables of the same quantity as the argument of the function, $x$. It should be noted that this does not necessarily have to be the case, because the exponent can be a variable of an entirely different quantity.

\subsubsection{Function $\cos \left\{1 /\left[x^{\cos (x)}\right]\right\}$}

This function is a representative of the family of functions $f\left(x^{y}\right)$, where $y$ is a negative variable. The function $\cos \left\{1 /\left[x^{\cos (x)}\right]\right\}$ is plotted in Figure 20. For the function $\cos \left\{1 /\left[x^{\cos (x)}\right]\right\}$, the argument of the function contains the quantity $1 / x$ to a variable power. The power $y$ is the function $\cos (x)$, which 
oscillates between the values 1 and -1 (Figure 2). Thus, the function $\cos \left\{1 /\left[x^{\cos (x)}\right]\right\}$ can take on values between $\cos (1 / x)$ where $y=1$ and $\cos (x)$ where $y=-1$.

For very small values of $x$, the power $\cos (x)$ is close to 1 so that the function $\cos \left\{1 /\left[x^{\cos (x)}\right]\right\}$ behaves much like $\cos (1 / x)$. This similarity is seen in Figure 20 as rapid oscillations as $x \rightarrow 0$.

As $x$ increases, the exponent $\cos (x)$ approaches 0 at $x=\pi / 2$. At this value of $x$, the function equals $\cos (1 / 1)$ or the cosine of 1 radian $(\sim 0.54)$. As $x$ increases further, $\cos (x)$ attains the value of -1 at $x=\pi$. At this point the function $\cos \left\{1 /\left[x^{\cos (x)}\right]\right\}$ behaves as $\cos (x)$, which has a value of -1 at $x=\pi$. This behavior is clearly seen in Figure 20 .

At $x=2 \pi$, the exponent $\cos (x)$ has value of 1 , so that the function $\cos \left\{1 /\left[x^{\cos (x)}\right]\right\}$ again behaves as $\cos (1 / x)$, which has a value of $\sim 1$ at $x=2 \pi$. In fact, at every point greater than $x=\sim \pi$, the function $\cos (1 / x)$ has a value $\sim 1$, as seen from Figure 4. Thus, the function $\cos \left\{1 /\left[x^{\cos (x)}\right]\right\}$ will have a value of $\sim 1$ at every point where the exponent is 1 . The exponent, $\cos (x)$, has a value of 1 at $x=0$, $2 \pi, 4 \pi \ldots(2 m \pi)$. Figure 20 shows that the function $\cos \left\{1 /\left[x^{\cos (x)}\right]\right\}$ has a value of 1 at $x=2 \pi, 4 \pi$, $6 \pi \ldots(2 m \pi)$.

In addition, oscillations between values of 1 and -1 are seen between the plateaus at $x=2 \pi, 4 \pi$, $6 \pi \ldots(2 m \pi)$. These oscillations are evident as sets of $1,3,5,7$ valleys, etc. The first of these, which contains only one valley, was explained previously for the first point at which $\cos (x)$ in the exponent has a value of -1 . The second set of oscillations, which contains three valleys, occurs around the point $x=3 \pi$. At the point $x=3 \pi$, the exponent $\cos (x)$ has a value of -1 . Thus, the function $\cos \left\{1 /\left[x^{\cos (x)}\right]\right\}$ again behaves as $\cos (x)$, which has a value of -1 at $x=3 \pi$. For each value $x=(2 m+1) \pi$, the function $\cos \left\{1 /\left[x^{\cos (x)}\right]\right\}$ has a value of -1 , which explains the central valley in each set.

The valleys surrounding the central one of each set can be explained as follows. Let the function $\cos \left\{1 /\left[x^{\cos (x)}\right]\right\}$ pass through the range $x=2 \pi$ to $4 \pi$. In this range, the function $\cos (x)$ has a value of 1 at $x=2 \pi$ and $4 \pi$; of 0 at $x=5 \pi / 2$ and $7 \pi / 2$; and of -1 at $x=3 \pi$. Therefore, the function $\cos \left\{1 /\left[x^{\cos (x)}\right]\right\}$ will take the form $\cos (1 / x)$ at $x=2 \pi$ and $4 \pi$ and $\cos (x)$ at $x=3 \pi$, as stated previously. At intermediate points, the function $\cos \left\{1 /\left[x^{\cos (x)}\right]\right\}$ somewhere takes forms such as $\cos \left(1 / x^{0.9}\right), \cos \left(1 / x^{0.8}\right) \ldots \cos \left(1 / x^{0.2}\right), \cos \left(1 / x^{0.1}\right), \cos \left(1 / x^{0}\right), \cos \left(1 / x^{-0.1}\right), \cos \left(1 / x^{-0.2}\right) \ldots \cos \left(1 / x^{-0.8}\right)$, $\cos \left(1 / x^{-0.9}\right),\left[\cos \left(1 / x^{-1}\right)\right], \cos \left(1 / x^{0.9}\right), \cos \left(1 / x^{0.8}\right) \ldots \cos \left(1 / x^{-0.2}\right), \cos \left(1 / x^{0.1}\right), \cos \left(1 / x^{0}\right), \cos \left(1 / x^{0.1}\right)$, $\cos \left(1 / x^{0.2}\right) \ldots \cos \left(1 / x^{0.8}\right), \cos \left(1 / x^{0.9}\right)$. Thus, the function should have the value of $\cos (1)$ or $\sim 0.54$ at $5 \pi / 2$ and $7 \pi / 2$. This can be seen to occur in Figure 20 , where these values are reached on the outermost walls of the outermost valleys of the set in this range. It is worth mentioning that, for the other sets at larger $x$ values, the outermost walls of the outermost valleys of those sets also contain the points corresponding to $(2 m+1) \pi / 2$. In other words, the oscillations in each set occur within the window bounded by the points $(4 m+1) \pi / 2$ and $(4 m+3) \pi / 2$. Closer inspection reveals that there is a regular series of points $(2 m+1) \pi / 2$ for which the value of the function is always $\cos (1)$.

Hence, the oscillations arise owing to the behavior of the function $\cos \left\{1 /\left[x^{\cos (x)}\right]\right\}$ within the range $x=5 \pi / 2$ to $7 \pi / 2$. Within this range, the function takes forms such as $\cos \left(1 / x^{-0.1}\right)$, $\cos \left(1 / x^{0.2}\right) \ldots \cos \left(1 / x^{0.8}\right), \cos \left(1 / x^{-0.9}\right), \cos \left(1 / x^{1}\right), \cos \left(1 / x^{-0.9}\right), \cos \left(1 / x^{0.8}\right) \ldots \cos \left(1 / x^{-0.2}\right), \cos \left(1 / x^{-0.1}\right)$, which are equivalent to $\cos \left(x^{0.1}\right), \cos \left(x^{0.2}\right) \ldots \cos \left(x^{0.8}\right), \cos \left(x^{0.9}\right), \cos (x), \cos \left(x^{0.9}\right), \cos \left(x^{0.8}\right) \ldots \cos \left(x^{0.2}\right)$, $\cos \left(x^{0.1}\right)$. Simultaneously with these changes in the exponent, the value of $x$ is constantly increasing. 
Also, since the function is fairly symmetric around the point $x=3 \pi$, only one-half of the range need be considered. Because the function acquires the values 1 and -1 in the range $x=5 \pi / 2$ to $3 \pi$, there must be points at which the argument has values of $2 m \pi$ and $(2 m+1) \pi$. Similarly, in the range $x=9 \pi / 2$ to $5 \pi$, there are two points each at which the argument acquires the values 1 and -1 ; in the range $x=13 \pi / 2$ to $7 \pi$, three points each; etc. The va'ues of these points could be found by plotting the function $1 / x^{\cos (x)}$ for each of these ranges. Indeed, such a plot shows that there is one point in the range $x=5 \pi / 2$ to $3 \pi$ at which the function $1 / x^{\cos (x)}$ acquires the value of $2 \pi$ and one point with the value of $\pi$. It is interesting to note that the function peaks at a value of $(3 \pi, 3 \pi)$. In fact, the function $1 / x^{\cos (x)}$ exhibits peaks at coordinates $(\pi, \pi),(3 \pi, 3 \pi),(5 \pi, 5 \pi)$, etc. Thus, at larger values of $x$, one finds no points corresponding to the values $2 m \pi$ or $(2 m+1) \pi$ for the peak at $(\pi, \pi)$ (except for the trivial instance of $m=0)$, one point each for each side of the peak at $(3 \pi, 3 \pi)$, two points each for each side of the peak at $(5 \pi, 5 \pi)$, etc. This observation readily explains, the oscillations within the windows from $(4 m+1) \pi / 2$ to $(4 m+3) \pi / 2$.

\subsubsection{Function $\cos \left\{1 /\left[x^{\sin (x)}\right]\right\}$}

This function is another representative of the family $f\left(x_{i} y\right)$, where $y$ is a negative variable. The function $\cos \left\{1 /\left[x^{\sin (x)}\right]\right\}$ is plotted in Figure 21. The behavior of this function is analogous to that of $\cos \left\{1 /\left[x^{\cos (x)}\right]\right\}$ in that it contains a series of sets of valleys separated by regions in which the function has a value of 1 . As before, the number of valleys in each set increases as $x$ increases.

The regions where the function has a value of 1 correspond to those regions where the function behaves as $\cos (1 / x)$ for $x>\sim \pi$. These regions are those where $\sin (x)$ has a value of 1 , or $\pi / 2$, $5 \pi / 2 \ldots(4 m+1) \pi / 2$. These regions can be clearly seen in Figure 21 .

As for $\cos \left\{1 /\left[x^{\cos (x)}\right]\right\}$, a regular series of points where the function $\cos \left\{1 /\left[x^{\sin (x)}\right]\right\}$ has the value $\cos (1)$ can be seen in Figure 21. These points occur where $\sin (x)=0$, which correspond to those points at $x=0, \pi, 2 \pi \ldots(m \pi)$.

The sets of valleys occur within the windows $(2 m+1) \pi$ to $(2 m+2) \pi$. In contrast with the function $\cos \left\{1 /\left[x^{\cos (x)}\right]\right\}$, the function $\cos \left\{1 /\left[x^{\sin (x)}\right]\right\}$ exhibits sets of valleys in which the central point of the function has a value of 0 . There are also points at which the function has values of 1 and -1 (although there are no points at which the function has a value of 1 for the range $x=\pi$ to $2 \pi$ ). There are always two more points at which the function has a value of -1 than at which it has a value of 1 . Thus, for the range $x=\pi$ to $2 \pi$, there two points at which the function has a value of -1 and one point at which it has a value of 0 ; for $x=3 \pi$ to $4 \pi$, four points at which it has a value of -1 , two points at which it has a value of 1 , and one point at which it has a value of 0 ; etc.

These oscillations can be explained in much the same way as was done for the function $\cos \left\{1 /\left[x^{\cos (x)}\right]\right\}$. Thus, a plot of $1 / x^{\sin (x)}$ exhibits peaks at $(3 \pi / 2,3 \pi / 2),(7 \pi / 2,7 \pi / 2)$, etc. For the peak values of $1 / x^{\sin (x)}$, the value of the function $\cos \left\{1 /\left[x^{\sin (x)}\right]\right\}$ is 0 , because $\sin (x)=-1$ for $(2 m+3) \pi / 2$, which gives $\cos (x)$ at these points; and $\cos (x)=0$ for $(2 m+1) \pi / 2$, which includes all points $(2 m+3) \pi / 2$. 'The function $\cos \left\{1 /\left[x^{\sin (x)}\right]\right\}$ will have values of 1 for each point at which $1 / x^{\sin (x)}$ $=2 m \pi$ and values of -1 for each point at which $1 / x^{\sin (x)}=(2 m+1) \pi$. It can be demonstrated easily that in the plot of $1 / x^{\sin (x)}$ there is one point on the ascending side of the peak at $3 \pi / 2$ at which the function has a value of $\pi$, which will give a value of -1 for the function $\cos \left\{1 /\left[x^{\sin (x)}\right]\right\}$; for the peak 
at $7 \pi / 2$, two points at which the function has values of $\pi$ and $3 \pi$, respectively, and one point at which the function has a value of $2 \pi$; etc. These observations readily explain the sets of valleys in the plot of the function $\cos \left\{1 /\left[x^{\sin (x)}\right]\right\}$.

\subsubsection{Function $\cos \left[x^{\cos (x)}\right]$}

This function is a representative of the family $f\left(x^{y}\right)$, where $y$ is a positive va-iable. The function $\cos \left[x^{\cos (x)}\right]$ is plotted in Figure 22. The behavior of this function is similar to that of the previous two functions in that it contains a series of sets of valleys separated by regions in which the function has a value of 1 . The number of valleys in each set increases as $x$ increases.

The regions in which the function $\cos \left[x^{\cos (x)}\right]$ has a value of 1 correspond to those regions for which $\cos (x)=-1$, or $(2 m+1) \pi$, for $x>\sim \pi$. Thus, regions in which the function $\cos \left[x^{\cos (x)}\right]$ has a value of 1 are seen near $x=\pi, 3 \pi, 5 \pi$, and so on.

The sets of valleys for this function lie between points with $x$ from $(2 m+1) \pi / 2$ to $(2 m+3) \pi / 2$. Thus, the first set of valleys occurs in he range of $x$ from $3 \pi / 2$ to $5 \pi / 2$; the second set in the range of $x$ from $7 \pi / 2$ to $9 \pi / 2$, etc. The sets of valleys have central points at which the function $\cos \left[x^{\cos (x)}\right]$ has a value of 1 . The number of points at which the function $\cos \left[x^{\cos (x)}\right]$ has a value of 1 is always one less than that at which it has a value of -1 for each set of valleys.

As before, these oscillations in the value of the function $\cos \left[x^{\cos (x)}\right]$ can be explained by examining a plot of the function $x^{\cos (x)}$. The plot of $x^{\cos (x)}$ exhibits peaks at coordinates $(2 \pi, 2 \pi)$, $(4 \pi, 4 \pi) \ldots(2 m \pi, 2 m \pi)$, in addition to a small peak at about $x=1.28$. For the peaks at $2 m \pi$, the function $\cos \left[x^{\cos (x)}\right]$ has a value of 1 , because $\cos (x)=-1$, which gives the function $\cos \left[x^{\cos (x)}\right]$ a value of $\cos (1 / x)$, and $\cos (1 / x)$ has a value of 1 at $x>\sim \pi$. For points on the peaks in the plot of $x^{\cos (x)}$ where the value is $\pi, 3 \pi \ldots(2 m+1) \pi$, the function $\cos \left[x^{\cos (x)}\right]$ will have a value of -1 . For points on the peaks in the plot of $x^{\cos (x)}$ where the value is $\pi, 2 \pi \ldots(2 m \pi)$, the function $\cos \left[x^{\cos (x)}\right]$ will have a value of 1 . Thus, the function $\cos \left[x^{\cos (x)}\right]$ exhibits oscillations that are consistent with the behavior of the function $x^{\cos (x)}$.

\subsubsection{Function $\cos \left[x^{\sin (x)}\right]$}

This is another representative of the family of functions $f\left(x^{y}\right)$, where $y$ is a positive variable. The function $\cos \left[x^{\sin (x)}\right]$ is plotted in Figure 23. The behavior is as expected and the explanation is left as an exercise for the reader.

\subsection{Functions $f^{n}\left(x^{a}\right)$}

Just as the common trigonometric functions can be raised to powers, the functions $f\left(x^{a}\right)$, where $a$ is a positive or negative constant or variable, also can be raised to powers. The following sections present examples of such operations. 


\subsubsection{Functions $f^{n}(x)$}

These are the powers of the common trigonometric functions such as $\sin ^{n}(x), \cos ^{n}(x), \tan ^{n}(x)$, $\cot ^{n}(x), \sec ^{n}(x)$, and $\csc ^{n}(x)$. Functions such as these are very well known and will not be treated further in this article.

\subsubsection{Functions $f^{n}\left(x^{a}\right)$}

\subsubsection{Functions $f^{n}\left(x^{n}\right)$, Where $n$ is an Integer}

The effect here is to convert all negative values of the functions to positive ones if $n$ is an even integer and to leave negative values of the functions unchanged if $n$ is an odd integer. In addition, there are subtle changes in the values of the functions that depend on the power to which the numbers less than or equal to 1 are raised.

To illustrate such an operation, the function $\cos ^{2}(1 / x)$ is plotted in Figure 24. A comparison with Figure 4 demonstrates that the value of the function $\cos ^{2}(1 / x)$ is everywhere positive or 0 whereas that for $\cos (1 / x)$ can be negative. It should be mentioned that the largest value of $x$ at which the function $\cos ^{2}(1 / x)$ peaks at a value of 1 is the same as that at which the function $\cos (1 / x)$ peaks at a value of -1 . Of course, the largest value of $x$ at which each function has a value of 0 is the same for both. However, for $\cos ^{2}(1 / x)$ this value represents a peak, whereas for $\cos (1 / x)$ this value is a point on a smooth curve.

For the remainder of the trigonometric functions, setting $n=2$ in the expressions $f^{n}\left(x^{a}\right)$, where $a=-1$, has the same effect of converting all negative values of the functions to positive values. For example, the functions $\tan ^{2}(1 / x)$ and $\cot ^{2}(1 / x)$ consist of a series of hyperboloids that have a value of 0 at and are undefined on either side of the low point. This result makes these two functions very similar to $\sec ^{2}(1 / x)$ and $\csc ^{2}(1 / x)$, which also consist of series of hyperboloids. However, the last two functions have a value of 1 at the low point.

Similar arguments apply to other functions $f^{n}\left(x^{a}\right)$ where $a$ is a negative number, for example, $\cos ^{2}\left(1 / x^{n}\right), \sin ^{2}\left(1 / x^{n}\right)$, and $\tan ^{2}\left(1 / x^{n}\right)$; and where $a$ is a positive number, for example, $\cos ^{2}\left(x^{n}\right)$ and $\tan ^{2}\left(x^{n}\right)$.

The behavior of functions $f^{n}\left(x^{a}\right)$, where $a$ is a positive or negative variable, is a little more complicated owing to the higher complexity of these functions. However, inspection is sufficient to show that negative values of these functions are converted to positive values if $n$ is an even integer.

\subsubsection{Functions $f^{n}\left(x^{n}\right)$, Where $n$ is a Number or Variable}

In addition to integral values, $n$ also can assume fractional or variable values. These functions will not be treated in this article. However, they must be mentioned, because they may be of use in fitting these functions to certain applications. 


\subsection{Sums of $f^{n}\left(x^{a}\right)$}

One of the most powerful aspects of these functions is their ability to be summed (added or subtracted) in order to produce functions that little resemble the relatively regularly changing components that they comprise. This feature will be illustrated using two examples.

\subsubsection{Function $\cos (1 / x)+\cos \left(1 / x^{2}\right)$}

The function $\cos (1 / x)+\cos \left(1 / x^{2}\right)$ is plotted in Figure 25. Beyond this range the function is of little interest, because it soon reaches a value of $\sim 2$ and remains constant at that value as $x \rightarrow \infty$. However, in the range $x=0$ to 1 , the functions $\cos (1 / x)$ and $\cos \left(1 / x^{2}\right)$ are "out of sync" to the extent that at some points the values sum to 0 and at others to nearly 2 or -2 . A comparison of Figure 25 with Figures 4 and 12 clearly reveals the complexity that can be introduced by summing just these two simple functions. Obviously, sums of more than two functions can readily be found. The complexity introduced by such a summation is quite astounding.

\subsubsection{Function $\cos \left(1 / x^{2}\right)+\cos \left(x^{2}\right)$}

This second example was chosen to illustrate an instance where the components of the sum are somewhat more obvious. The function $\cos \left(1 / x^{2}\right)+\cos \left(x^{2}\right)$ is plotted in Figure 26. An inspection of Figures 12 and 17 shows that the function $\cos \left(1 / x^{2}\right)$ has a value of 1 for $x>-1$, whereas the function $\cos \left(x^{2}\right)$ has a value close to 1 for $x<\sim 0.5$. Thus, when these two functions are summed, the oscillations for $x<\sim 0.5$ are more or less controlled by $\cos \left(1 / x^{2}\right)$; those at $x>\sim 1$ are controlled by $\cos \left(x^{2}\right)$. Therefore, the plot of $\cos \left(1 / x^{2}\right)+\cos \left(x^{2}\right)$ looks very similar to that of the former for $x<\sim 0.5$ and to that of the latter for $x>\sim 1$.

\subsubsection{Other Sums}

Any number or type of functions can be summed. For example, consider the plots shown in Figures 20 and 22. It is interesting that the valleys in one of these plots occur at values where the other plot has a value close to 1 . Thus, if these two functions are summed, the result is a series of sets of valleys in which the number of valleys in each set increases by one from each set to the next.

Summation of the functions shown by the plots in Figures 20 and 21 would give rise to a very interesting series of sets of valleys, because the sets in the two plots are offset by a factor of $\pi / 2$.

The variety of other sums that can be envisioned is limited only by the imagination of the person composing the them.

\subsection{Products of $f^{n}\left(x^{a}\right)$}

Another powerful aspect of these functions is their ability to be multiplied or divided by one another in order to produce functions that little resemble the relatively regularly changing components that they comprise. This feature will be illustrated using the following example. 


\subsubsection{Function $\cos (1 / x) \cdot \cos \left(1 / x^{2}\right)$}

The function $\left[\cos (1 / x) \cdot \cos \left(1 / x^{2}\right)\right]$ is plotted in Figure 27. An inspection of Figures 4 and 12 shows, as before, that the two functions $\cos (1 / x)$ and $\cos \left(1 / x^{2}\right)$ are out of sync in this range. Thus, at some points the former is positive whereas the latter is negative and vice versa. An interesting feature of this plot occurs as the function approaches 1 . Because $\cos (1 / x)$ passes through 0 for the last time at a smaller value of $x$ than $\cos \left(1 / x^{2}\right)$, the product of these two functions passes through 0 at $x=2 / \pi$ and $(2 / \pi)^{1 / 2}$. Because the function $\cos \left(1 / x^{2}\right)$ is negative until it reaches $(2 / \pi)^{1 / 2}$, the product is also negative between the points $x=2 / \pi$ and $(2 / \pi)^{1 / 2}$. This result can be seen clearly in Figure 27 .

\subsubsection{Other Products}

Like for the sums of these functions, products of any of these functions also can be constructed. Several interesting possibilities should be apparent to the reader by this time.

\subsection{Functions $f\left(a^{x}\right), f\left(a^{-x}\right), f\left(y \cdot a^{x}\right)$ and $f\left(y \cdot a^{-x}\right)$}

These functions involve nonlinear arguments such as $a^{x}$, where $a$ is a constant, and $y \cdot a^{x}$, where $y$ is a variable.

\subsubsection{Functions $f\left(a^{2}\right)$}

The trigonometric functions of $a^{x}$, where $a$ is a constant, display behavior similar to that of the functions $f\left(x^{a}\right)$. This behavior is readily evident because in both instances the argument of the function is an exponential. For example, a plot of $2^{x}$ has essentially the same shape as that of $x^{2}$. Thus, the trigonometric functions $f\left(a^{\mathfrak{x}}\right)$ give plots similar to those of $f\left(x^{a}\right)$.

\subsubsection{Functions $f\left(a^{-n}\right)$}

These functions are relatively well-behaved as far as the trigonometric functions of nonlinear quantities are concerned. Because $a^{-x}$ has a value of 1 at $x=0$, the trigonometric functions have the appropriate value at $x=0$; for example, $\cos (1)=\sim 0.54$. Thus, there are no oscillations as $x \rightarrow 0$. At the other extreme, because $a^{-x}$ approaches 0 as $x \rightarrow \infty$, the trigonometric functions approach the appropriate value as $x \rightarrow \infty$; for example, $\cos (0)=1, \sin (0)=0$, etc.

\subsubsection{Functions $f\left(y \cdot a^{\mathfrak{n}}\right)$}

A variety of forms is possible for these functions. For the sake of illustration, consider the argument $\mathrm{e}^{x / x}\left(a^{x}=\mathrm{e}^{x}\right.$ and $\left.y=1 / x\right)$. The argument approaches a value of $\infty$ as $x \rightarrow 0$ and a value of $\infty$ as $x \rightarrow \infty$. There is a large region around 1 where the argument has a value in the neighborhood of $\mathrm{e}$ (the value is e exactly at $x=1)$. Thus, the function $\cos \left(\mathrm{e}^{x} / x\right)$ oscillates rapidly as $x \rightarrow 0$ and as $x \rightarrow \infty$ and has a region near $x=1$ where the value is in the neighborhood of -1 , because $\mathrm{e}$ is only slightly less than $\pi$. 


\subsubsection{Functions $f\left(y \cdot a^{-x}\right)$}

As an illustration of these functions, consider the argument $x / \mathrm{e}^{x}\left(\mathrm{e}^{-x}=a^{-x}\right.$ and $\left.y=x\right)$. The plot of $\cos \left(x / \mathrm{e}^{x}\right)$ is interesting in that it has a value of 1 at $x=0$ and reaches a minimum of $\sim 0.93$ at $x=1$ $\left(1 / \mathrm{e} \sim 0.368\right.$, which represents a peak in the plot of $\left.x / \mathrm{e}^{\mathfrak{x}}\right)$. Then $\cos \left(x / \mathrm{e}^{x}\right)$ again approaches a value of 1 as $x$ increases. The plot of this function is similar to that of the potential well seen in a plot of E vs. $r$ for a diatomic molecule with the exception that it starts at 1 and approaches 1 as $x \rightarrow \infty$. 


\subsection{Discussion}

\subsection{General}

The plots shown in this article are constructed using radians as the units for the argument of the trigonometric functions, because the plotting program is limited to assigning some physical quantity to it. Although the plots in general cover only positive values of $x$, it should be recognized that the plots would retain the symmetry of the parent function if plotted for negative values of $x$. Thus, plots of the cosine functions would be symmetric about the origin whereas those of the sine would be antisymmetric.

The results presented in Section 2.0 demonstrate that the common trigonometric functions can operate on quantities other than the linear ones. Indeed, such functions have been mentioned in calculus books as interesting problems in the differentiation and integration of trigonometric functions and expansions by Taylor's series. Thus, it has been shown quite convincingly that functions such as $1 / x^{a}$, $x^{a}$, and even $x^{f(x)}$ are suitably transformed by the common trigonometric functions. One way of ' isualizing this phenomenon is to consider that these functions nonlinearly generate radians as the argument of the trigonometric functions. It then becomes apparent why a function such as $\cos (1 / x)$ oscillates more and more rapidly as $x \rightarrow 0$. The value of the function increases exponentially, thereby producing increments of radians at an exponentially increasing rate.

The common trigonometric functions can be expressed in exponential form. For example, $\sin (x)=\left(\mathrm{e}^{\mathrm{ix}}-\mathrm{e}^{-i x}\right) / 2 i$ and $\cos (x)=\left(\mathrm{e}^{i x}+\mathrm{e}^{-i x}\right) / 2$, from which the exponential forms of all other common trigonometric functions can be derived. Thus, for a function such as $\cos (1 / x)$, the exponential form would be $\cos (1 / x)=\left(\mathrm{e}^{i / x}+\mathrm{e}^{-i / x}\right) / 2$. The plot of such an exponential function is expected to be identical to that of the trigonometric function. The corresponding equations can be derived for the other functions introduced in this article.

\subsection{Applications}

The functions introduced in this article may have application to several areas of science. These functions exhibit unique properties that in many instances match those of well-known physical phenomena. Some of the more significant possible applications are discussed below.

\subsubsection{Quantizing Factors}

The fact that many of the functions presented vary in value between -1 and 1 makes them attractive for use in quantum mechanics as quantizing factors for other functions. The following example illustrates this.

Consider the function $x^{2}\left[\cos \left(1 / x^{2}\right)\right]$. This function is plotted in Figures 28 through 30 for several ranges of $x$. In Figure 28, the plot appears more or less typical of a normal exponential relationship. However, very close inspection reveals that there is a slight anomaly at $x<1$. Therefore, the plot is 
shown in greater detail in Figure 29. It is quite clear from Figure 29 that the function $x^{2}\left[\cos \left(1 / x^{2}\right)\right]$ exhibits regular oscillations in the range $x<1$. Further expansion of the range (Figure 30) shows that these oscillations increase in frequency as $x \rightarrow 0$.

Thus, the effect of multiplying $x^{2}$ by the function $\cos \left(1 / x^{2}\right)$ is to superimpose on $x^{2}$ an oscillating or quantizing function. This function increases in frequency as $x \rightarrow 0$ such that by the time the range $x<1$ is reached the composite function bears no resemblance to the function $x^{2}$ but appears more like a damping function, with the exception that the frequency is constantly increasing as $x \rightarrow 0$.

Hence, it is interesting that the function $x[\cos (1 / x)]$ exhibits similar behavior. In fact, the plot of this function is, for all intents and purposes, a straight line for the range $x=0$ to $\infty$, with an anomaly seen from $x=0$ to 1 . Further inspection of this anomaly reveals the same oscillatory nature as seen for $x^{2}\left[\cos \left(1 / x^{2}\right)\right]$. The oscillations become very rapid for $x<0.12$. Again, the function $\cos (1 / x)$ can be considered a quantizing factor for the function $x$.

The significance of such a function rests in the analogy to quantum mechanics, wherein masses larger than a certain quantity have DeBroglie wavelengths that are imperceptibly small, whereas those smaller than a certain quantity have wavelengths that are significant in relation to the mass. The instantaneous frequency of the function at very small $x$ (where $x$ represents a mass) could be related to the DeBroglie wavelength of a certain mass. For example, the period of oscillation of a function such as $\cos (1 / x)$ is the range in which $x$ changes by a value of $2 \pi$. Thus, the range from $x=1 / \pi$ to $x=1 / 3 \pi$ represents one oscillation. This can be seen in Figure 4. Similarly, the range from $x=1 / 100 \pi$ to $\mathrm{x}=1 / 102 \pi$ also represents one oscillation. However, in the second instance the range of $x$ is from $3.183 \cdot 10^{-3}$ to $3.121 \cdot 10^{-3}$ whereas in the first the range of $x$ is from 0.3183 to 0.1061 . Thus, the "frequency" in the second instance is $1 /(3.813-3.121) \cdot 10^{-3}$, or $1.445 \cdot 10^{3}$ (per unit $x$ ), whereas it is $1 /(0.3183-0.1061)$, or 4.712 (per unit $x)$ in the first instance. By adjusting the power of $x$ in $\cos (1 / x)$ the frequency of the function can be controlled.

Also, the function is especially interesting because, if the quantizing factor $\cos (1 / x)$ is omitted, it bears a striking resemblance to many well-known equations, one of which could be $E=m c^{2}$, where $c^{2}$ is a constant, and $E$ is directly proportional to the mass. By superimposing the quantizing factor on this equation, the energy and mass become quantized.

Moreover, it is worth mentioning that whole families of functions $\cos \left(1 / x^{a}\right), \sin \left(1 / x^{a}\right), \tan \left(1 / x^{a}\right)$, etc., can be used as quantizing factors where quantization as $x \rightarrow 0$ is desired. Thus, depending on the physical system under study, the exponent $a$ can be adjusted to give a fit for any experimental data that may be available. As we have seen in the Results section, $a$ can even be a variable.

If quantization is desired as $x \rightarrow \infty$, then functions of the families $\cos \left(x^{a}\right), \sin \left(x^{a}\right), \tan \left(x^{a}\right)$, etc., can be used to produce the desired effect. The functions $\cos \left(x^{a}\right)$ are interesting, because they have values of 1 at $x=0$. The functions $\sin \left(x^{a}\right)$ are also interesting, because they have values of 0 at $x=0$.

\subsubsection{Gravity}

These quantizing factors may be applicable to equations that are known to describe the effects of gravity. For example, the equation for gravitational force, $F=-G \mathrm{~m}_{1} \mathrm{~m}_{2} / \mathrm{r}^{2}$, contains the three variables $\mathrm{m}_{1}, \mathrm{~m}_{2}$, and $r$. By fixing the origin of a coordinate system at the center of one of the masses, say 
$m_{1}$ for sake of argument, the gravitational force could be viewed as the interplay of $m_{2} / r^{2} v$ s. $m_{1}$. By superimposing one of the described quantizing factors on the equation for gravitational force, an equation that provides for quantized gravitational fields could be obtained. For example, suppose $F=\left(-G \mathrm{~m}_{1} \mathrm{~m}_{2}\right) \cdot\left(1 / \mathrm{r}^{2}\right)\left[\cos \left(x^{a}\right)\right]$, where $x$ is in some way dependent on $r$. The task would be to gither experimental data for appropriate systems and to adjust the values of $a$ and $x$ so that the function $\left(-G \mathrm{~m}_{1} \mathrm{~m}_{2}\right) \cdot\left(1 / \mathrm{r}^{2}\right)\left[\cos \left(x^{a}\right)\right]$ would exhibit peaks at the points where $\mathrm{r}$ corresponds to stable gravitational systems. Note that as $\mathrm{r} \rightarrow 0, F \rightarrow \infty$; and as $\mathrm{r} \rightarrow \infty, F \rightarrow 0$. In this instance, the quantizing factor that is desired is one that oscillates more and more rapidly as $x \rightarrow \infty$, for example, $\cos \left(x^{a}\right)$; the quantization would be superimposed on the function as $r$ increases whereas the function would be relatively unaffected at small $\mathrm{r}$, because $\cos \left(x^{a}\right)=1$ at $x=0$.

\subsubsection{Final Theory}

The unification of the forces of nature would represent a major milestone in developing a final theory of the universe. Previous efforts to identify equations that would accomplish this feat have led to quantum electrodynamics, special relativity, and string theory, among others. The functions presented in this article may be applicable to this problem, as indicated in the following discussion.

First, several of the functions presented in this article display properties that are compatible with the need to remain defined at very large and very small quantities. For example, the function $\cos (1 / x)$ approaches 1 as $x \rightarrow \infty$ and is reduced to what can be considered a frequency as $x \rightarrow 0$. Similarly, $\sin (1 / x)$ approaches 0 as $x \rightarrow \infty$ and also is reduced to what can be considered a frequency as $x \rightarrow 0$. These properties are very important to a final theory, because the inability to deal with the infinities that arise has been the stumbling block of many otherwise attractive theories.

Second, several of the functions exhibit discontinuities at regular intervals while remaining otherwise defined as $x \rightarrow 0$. Such functions would include $\tan (1 / x), \sec (1 / x)$, and $\cot ^{2}\left(1 / x^{2}\right)$, among others. This is consistent with the structure of matter, in which discontinuities are seen in the masses characteristic of the building blocks (plasma, atoms, quarks, etc.). A description of the structure of matter must be capable of treating such discontinuities.

Finally, if one considers that the universe is subject to attractive and repulsive forces, then it stands to reason that the existence or nonexistence of one type of matter or another depends on the balance between these two forces. This balance could possibly be expressed as the sum of two equations, one for the attractive force and one for the repulsive force acting on the particular matter. There are many examples where this balance of forces can be seen. Consider, for example, the following: Black holes, the densest members of the universe, attract matter into themselves and induce the matter to release radiation ( $\gamma$-radiation or radiation of even higher energy; for example, cosmic radiation) by causing the matter to become more dense under the extreme gravitation present (the increased attractive force in the matter is balanced by the release of radiation). Stars are massive enough that their gravitational field is sufficient to cause individual atoms to fuse into heavier nuclei, which release radiation and particles in the process (the increased attractive force in the nuclei resulting from the fusion is balanced by the release of radiation and particles). Nuclei of very heavy atoms build up so much repulsive force that they spontaneously fission into smaller fragments that are more stable (the increased repulsive force in the nuclei as the mass grows results in the release of smaller particles and radiation). Other examples can be cited that involve the reaction of chemical compounds, the decay of neutrons, and the instability of subatomic particles. The important point is that there are two kinds of forces in the 
universe, attractive and repulsive ones. A final theory could very well consist of an equation that is a composite of two functions that represent the attractive and repulsive forces acting on matter.

Several of the functions introduced in this article are prime candidates for representing these attractive and repulsive forces. The attractive force is expected to increase as the density of matter increases. The densest matter would be subject to the strongest quantization whereas the most diffuse matter would be more or less a continuous systen with little quantization. Thus, a function is sought that is quantized at large densities and more or less continuous at small densities. In this instance, a function such as $\sin \left(x^{a}\right)$, where $x$ depends in some way on the density and $a$ is positive, seems appropriate because the attractive force would be 0 at very low densities and would be quantized at high densities. The repulsive force also is expected to increase as the density of matter increases. However, in this instance a negative force is desired, because the force is repulsive in nature. In this instance also, the quantization is expected to be greatest at the highest densities. Thus, the function desired is similar in nature to that for the attractive force but of opposite sign. Clearly, a function such as $-\sin \left(x^{a}\right)$, where $x$ depends in some way on the density and $a$ is positive and different from $a$ for the attractive force, is suitable in this instance. Then, the task is to determine the values for the exponents in the equations for the attractive and repulsive forces that will produce a sum with peaks at points corresponding to densities at which stable forms of matter are observed. This could be done by fitting the equations to experimental data.

\subsection{Computing Needs}

The implementation of the functions introduced in this article will require several new computer programs to be written. These should address the following areas.

\subsubsection{Integration}

Computer programs that integrate the functions need to be written. These programs are needed so that the areas encompassed by the functions can be measured and quantified. The programis should be capable of integrating the functions over both positive and negative values of $x$. The programs also should be capable of integrating sums, products, and quotients of the functions.

\subsubsection{Curve Fitting}

Critical capabilities to the implementation of the functions introduced in this article are the abilities to deconvolute complex functions into components, and to adjust exponents and constant factors so that functions can be fitted to experimental data. Computer programs need to be written for this task. 


\subsection{Acknowledgments}

I would like to thank Dr. Gregg J. Lumetta and Mr. David L. Alexander of Pacific Northwest Laboratory (PNL) for kindly permitting me to use their equipment to generate the plots and perform the calculations and Catherine C. Lumetta of PNL for her editorial assistance. Dr. John A. Olson of Baylor University is to be commended for his helpful discussions during the formative stages of this work. I would also like to thank my wife and family for their support during the preparation of the manuscript. 


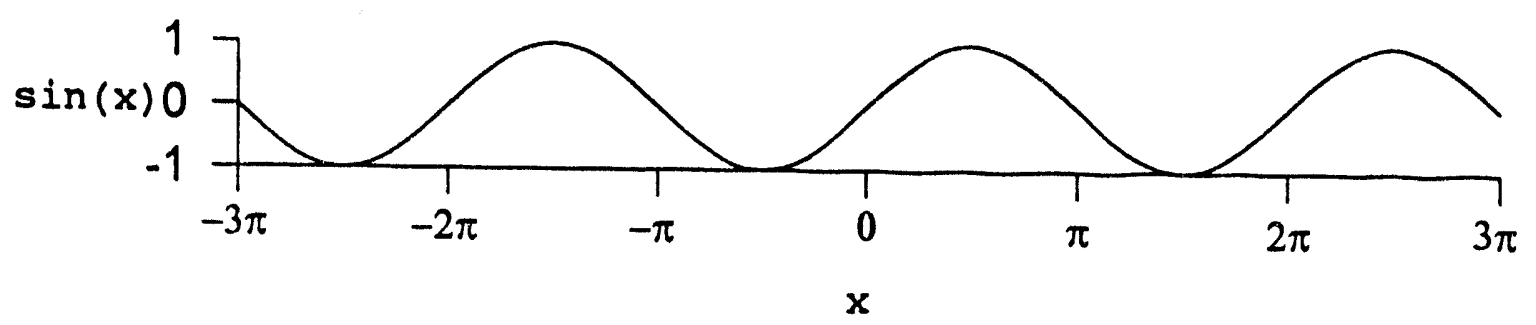

Figure 1. Plot of $\sin (x)$

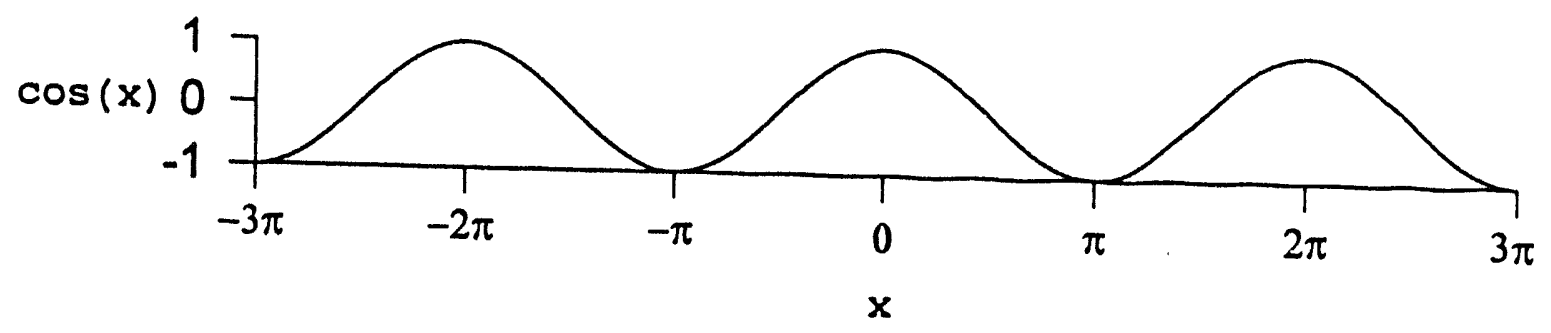

Figure 2. Plot of $\cos (\mathrm{x})$

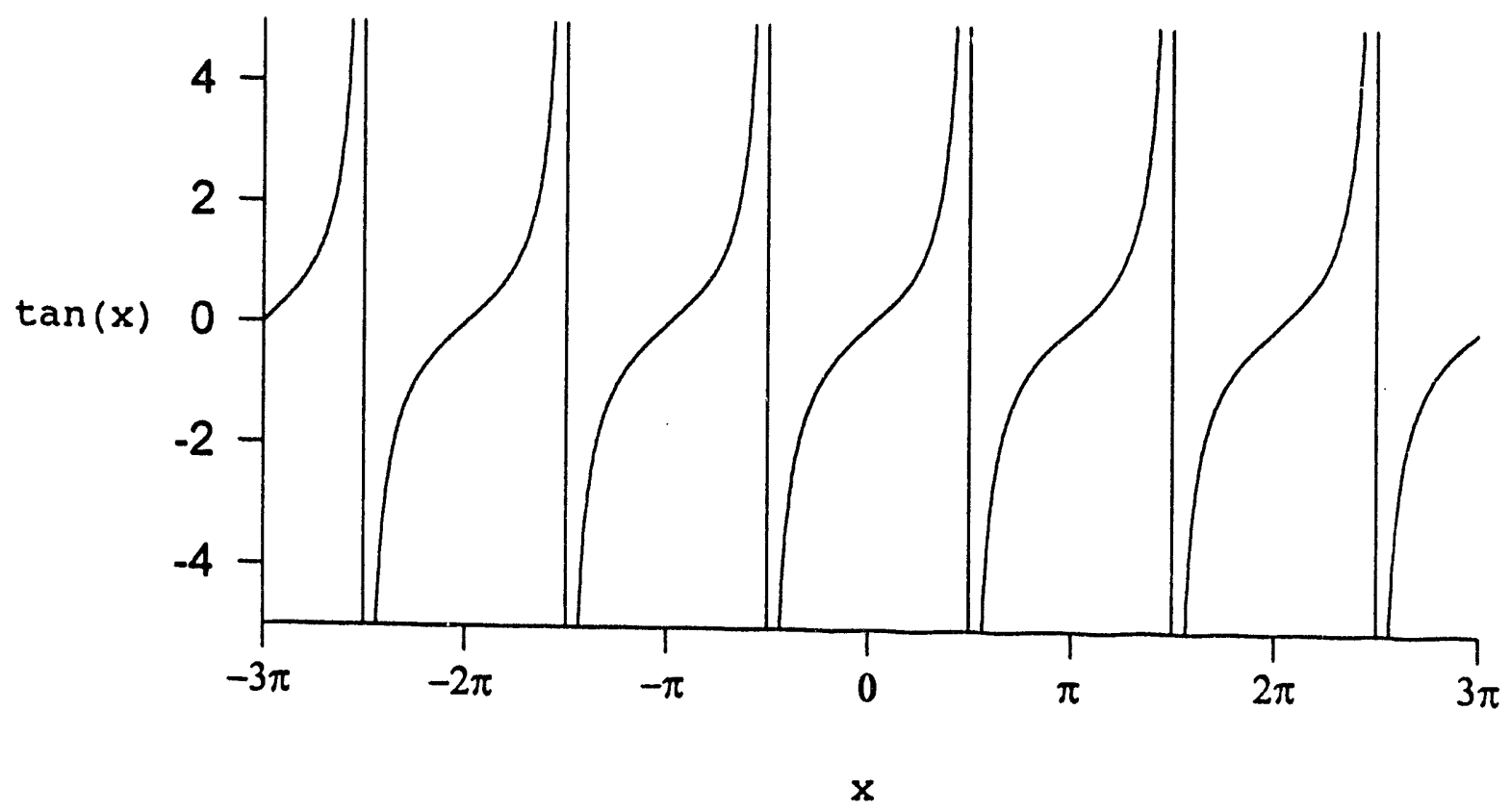

Figure 3. Plot of $\tan (\mathrm{x})$ 


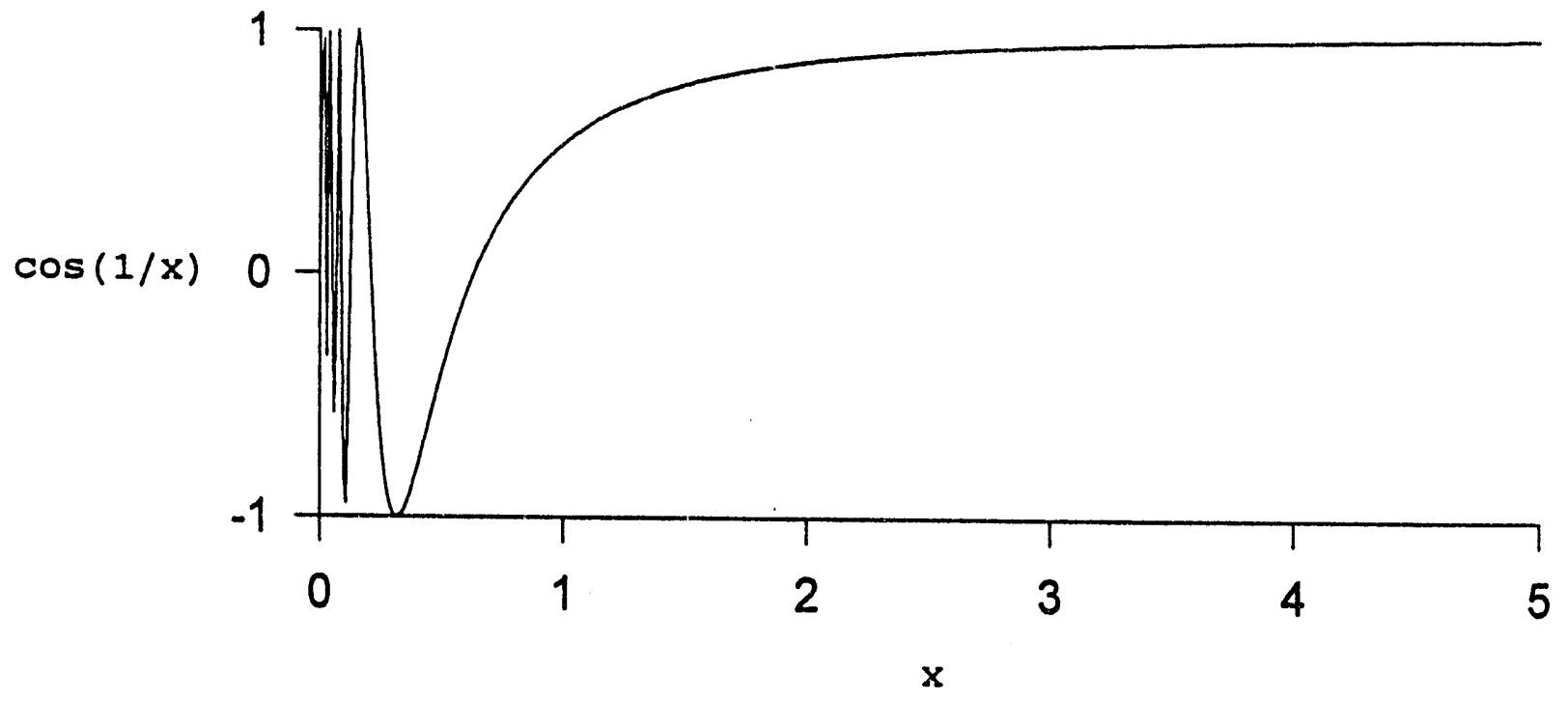

Figure 4. Plot of $\cos (1 / x)$ for $0<x<5$

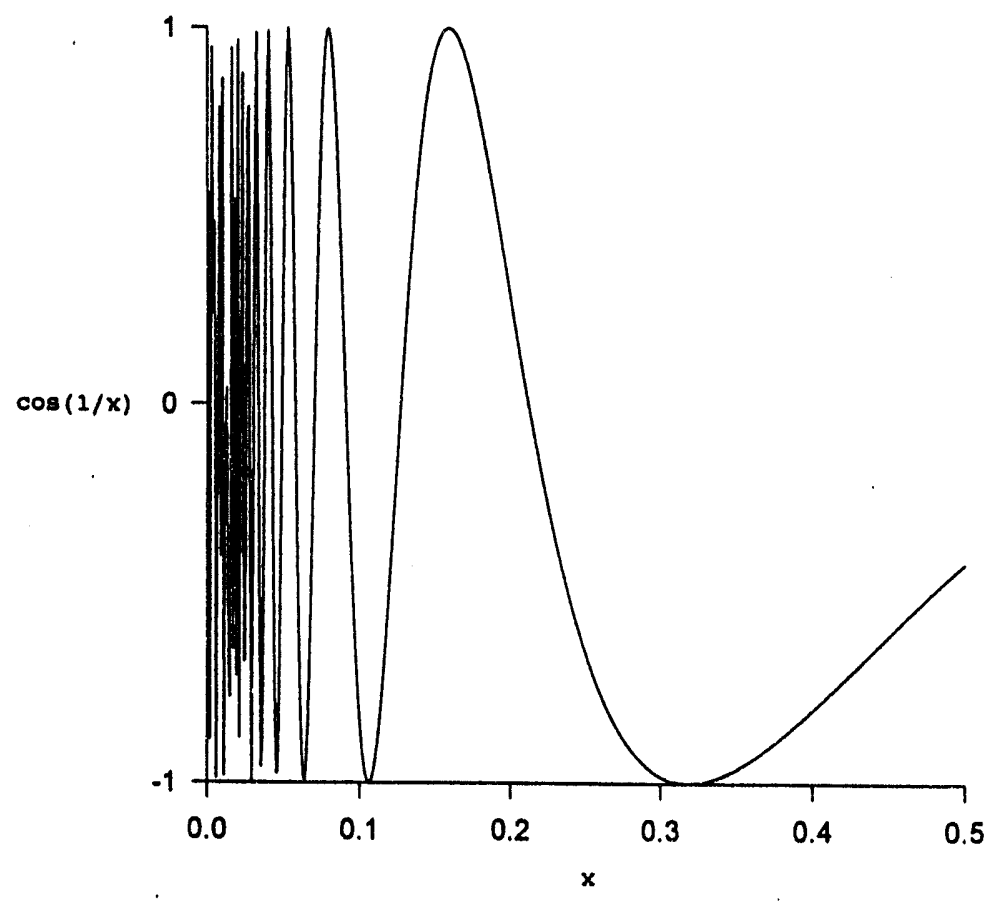

Figure 5. Plot of $\cos (1 / x)$ for $0<x<0.5$ (expanded along $x$ ) 


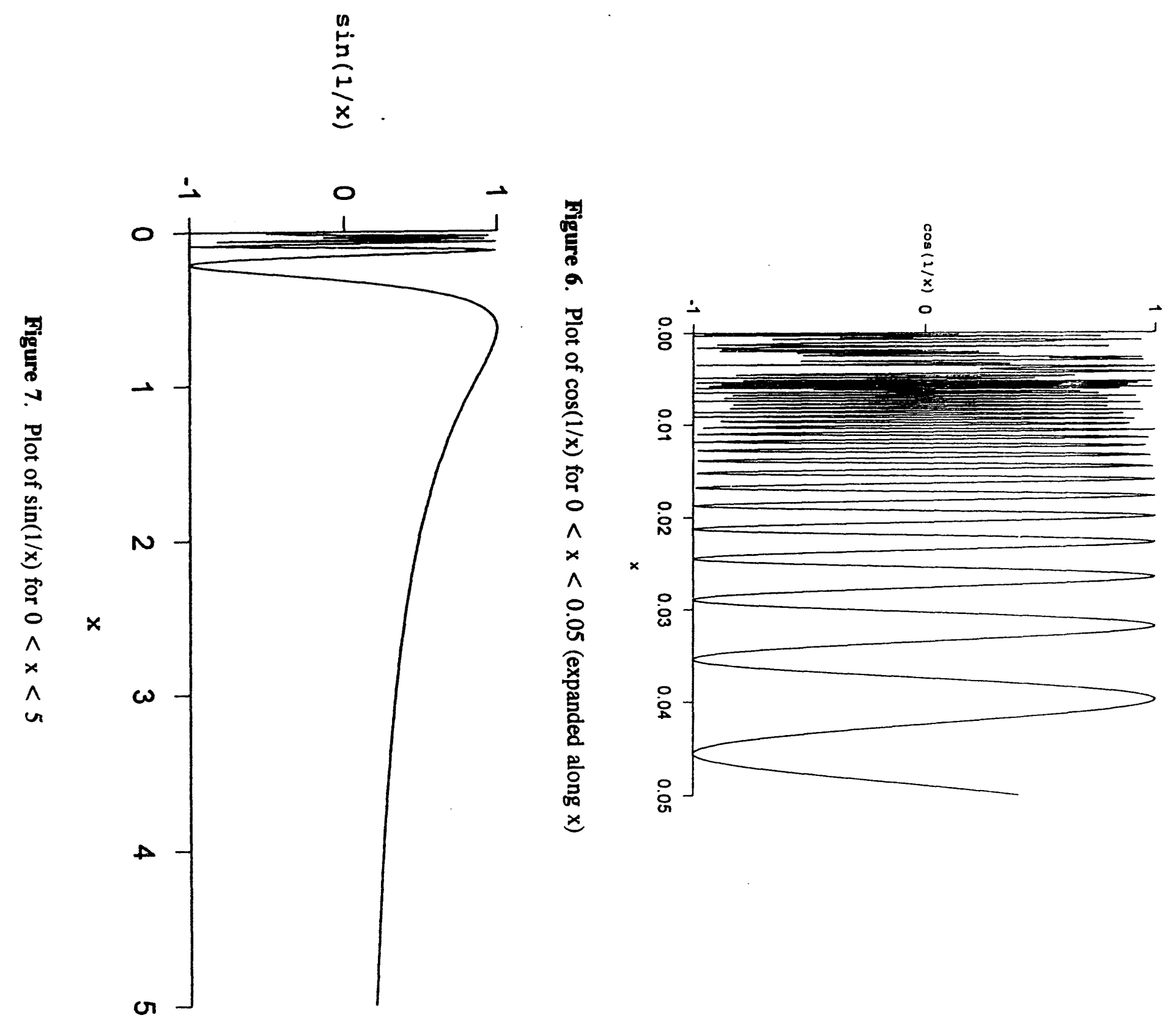




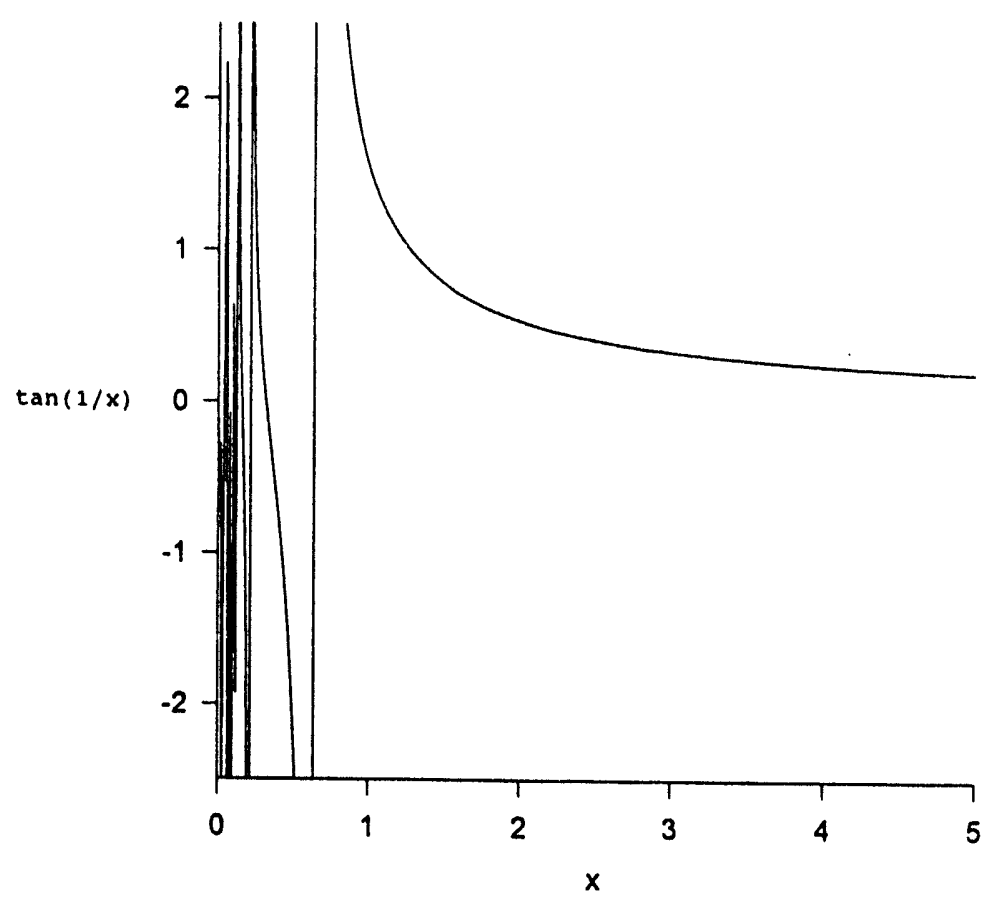

Figure 8. Plot of $\tan (1 / \mathrm{x})$ for $0<x<5$

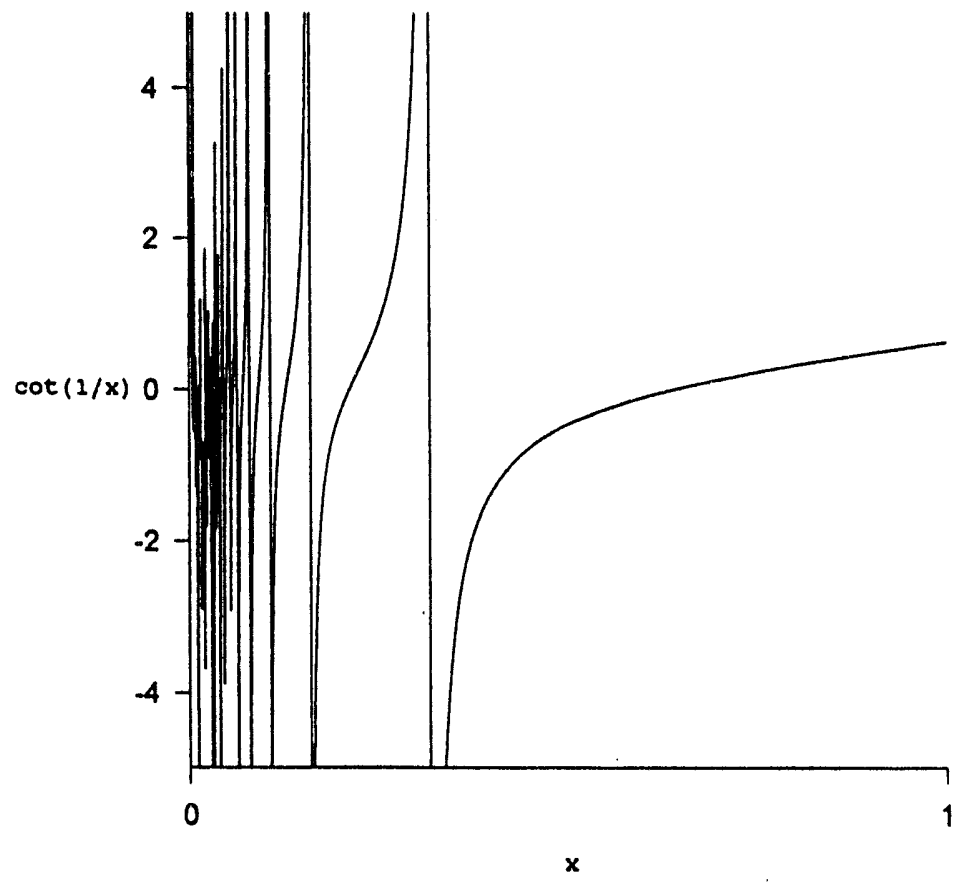

Figure 9. Plot of $\cot (1 / \mathrm{x})$ for $0<\mathrm{x}<1$ (expanded along $\mathrm{x}$ ) 


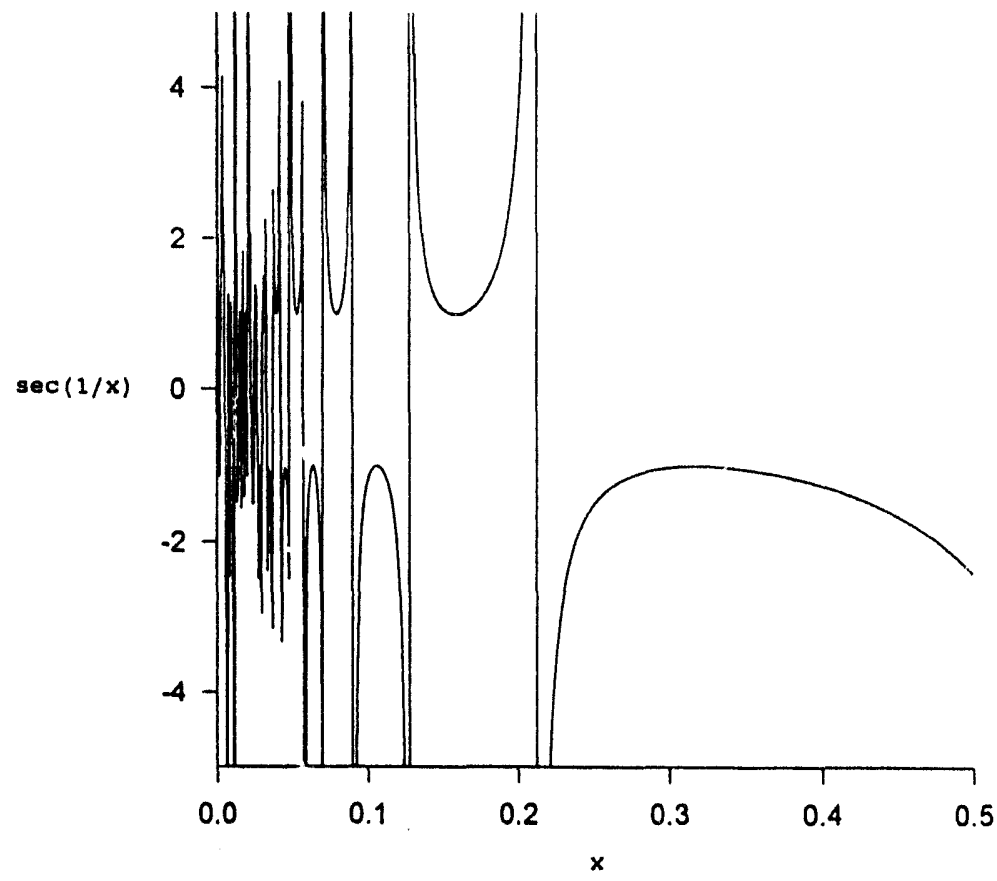

Figure 10. Plot of $\sec (1 / x)$ for $0<x<0.5$ (expanded along $x$ )

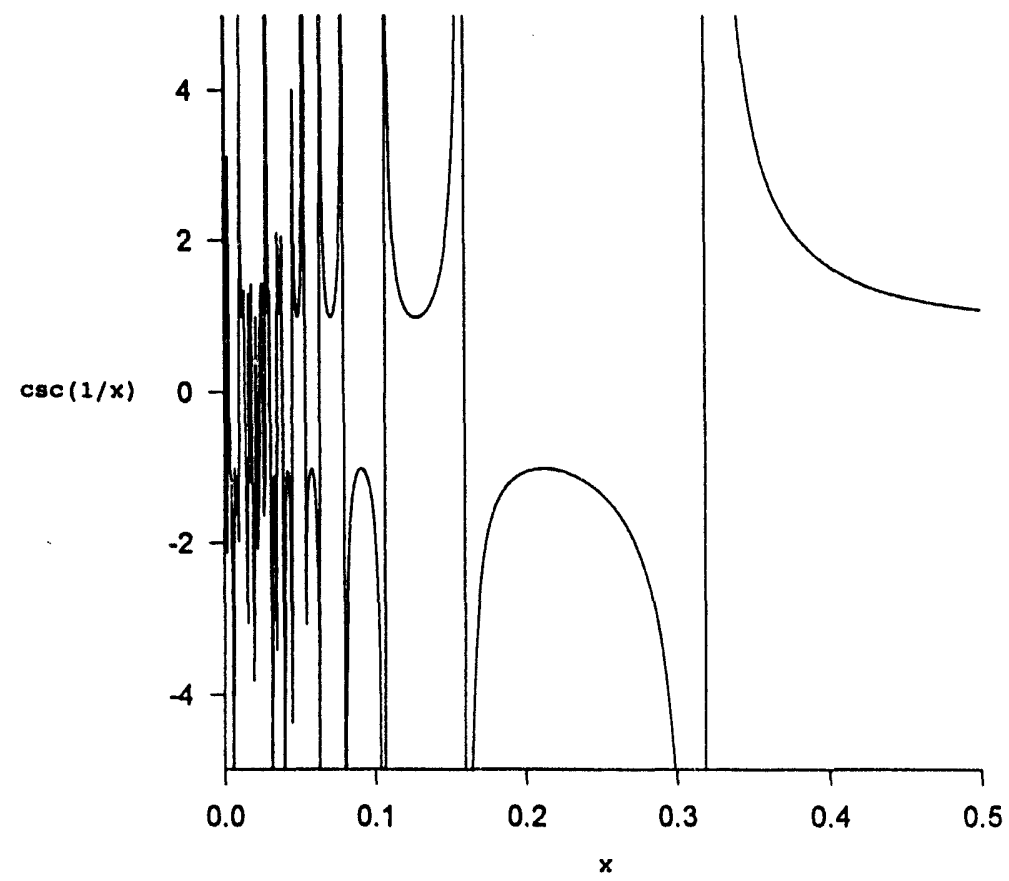

Figure 11. Plot of $\csc (1 / \mathrm{x})$ for $0<\mathrm{x}<0.5$ (expanded along $\mathrm{x}$ ) 


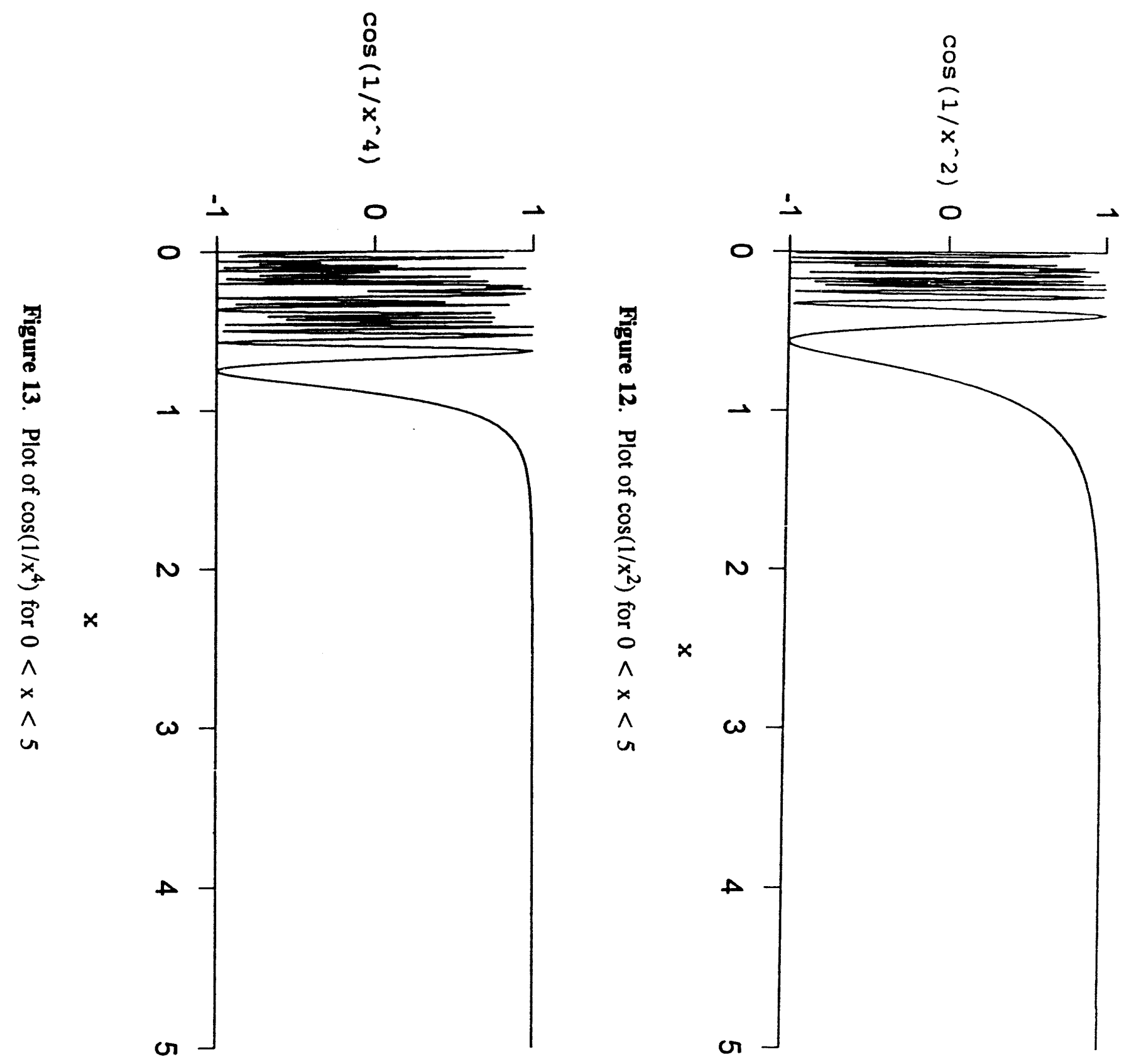




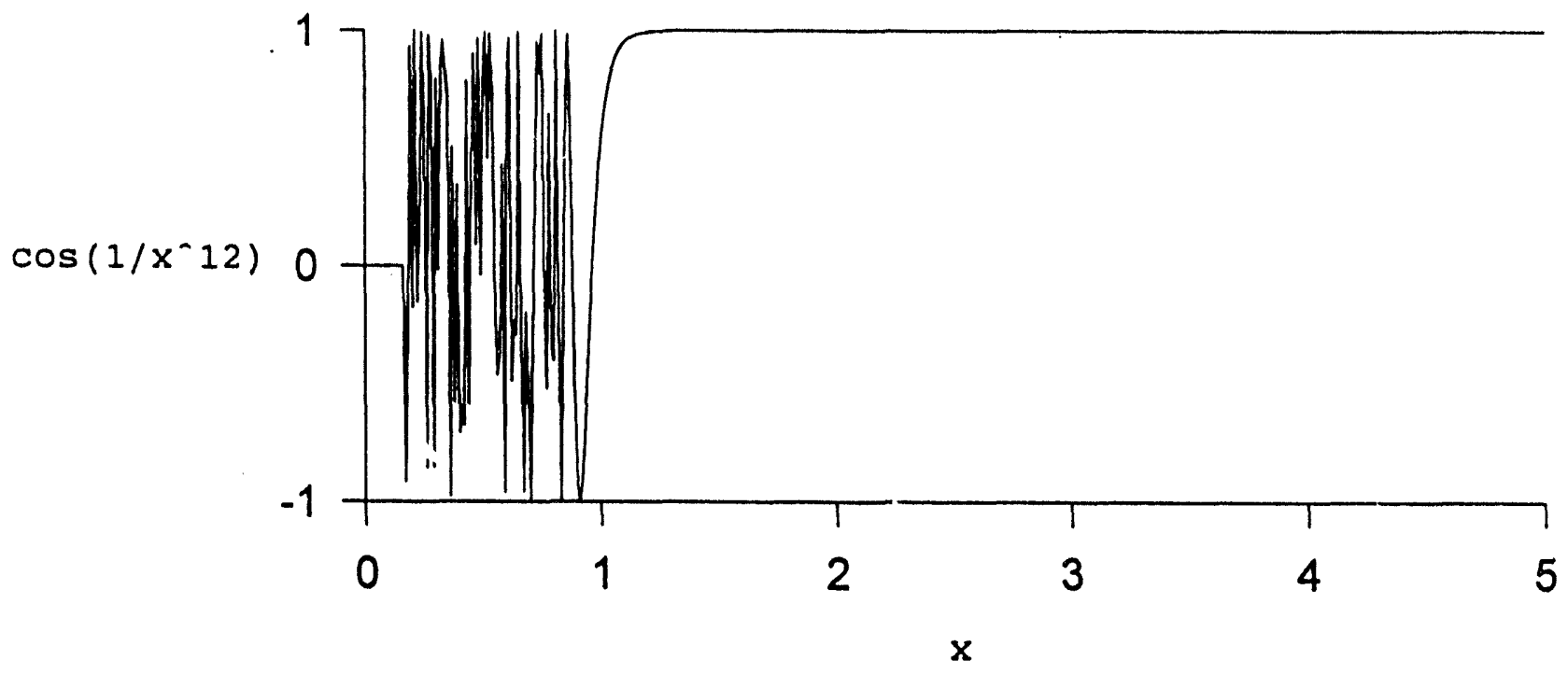

Figure 14. Plot of $\cos \left(1 / x^{12}\right)$ for $0<x<5$

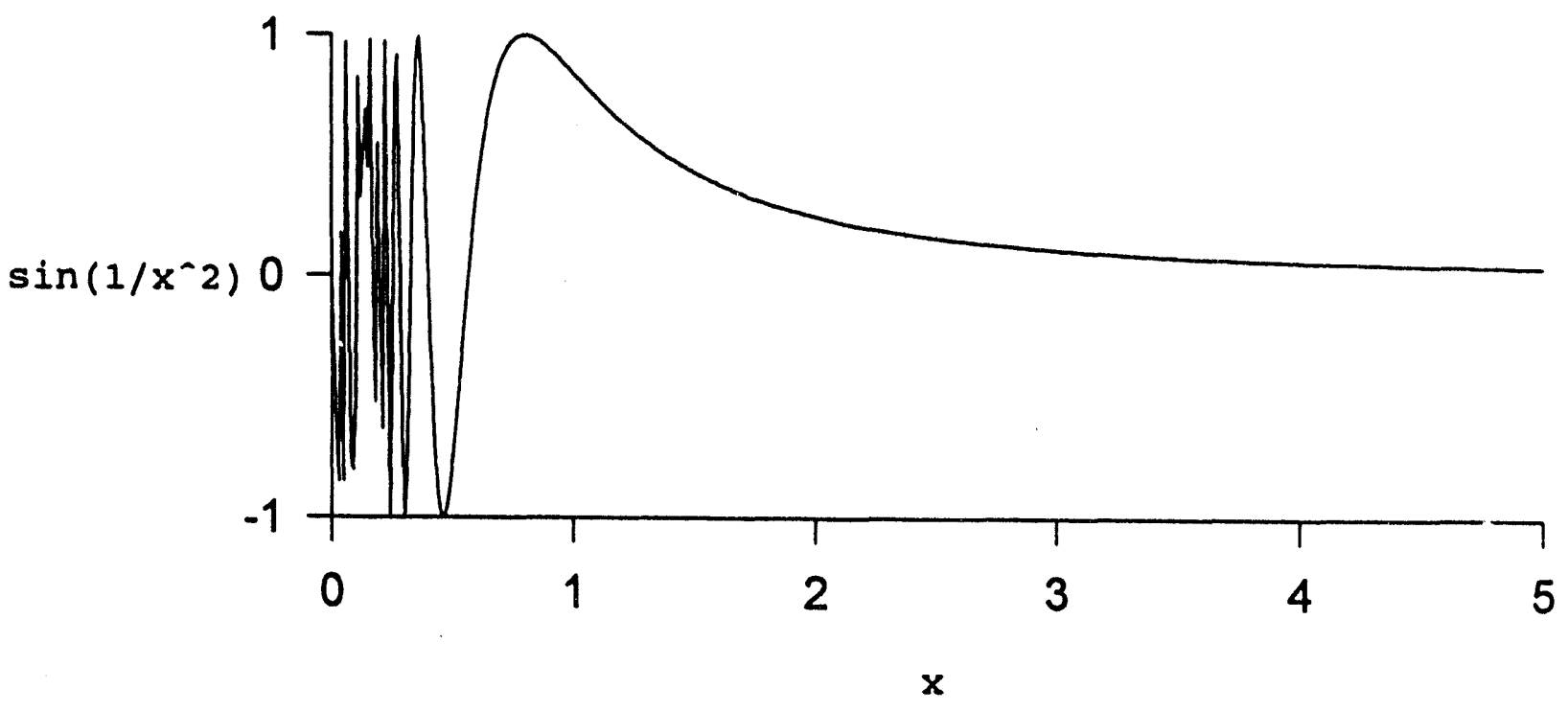

Figure 15. Plot of $\sin \left(1 / \mathrm{x}^{2}\right)$ for $0<\mathrm{x}<5$ 


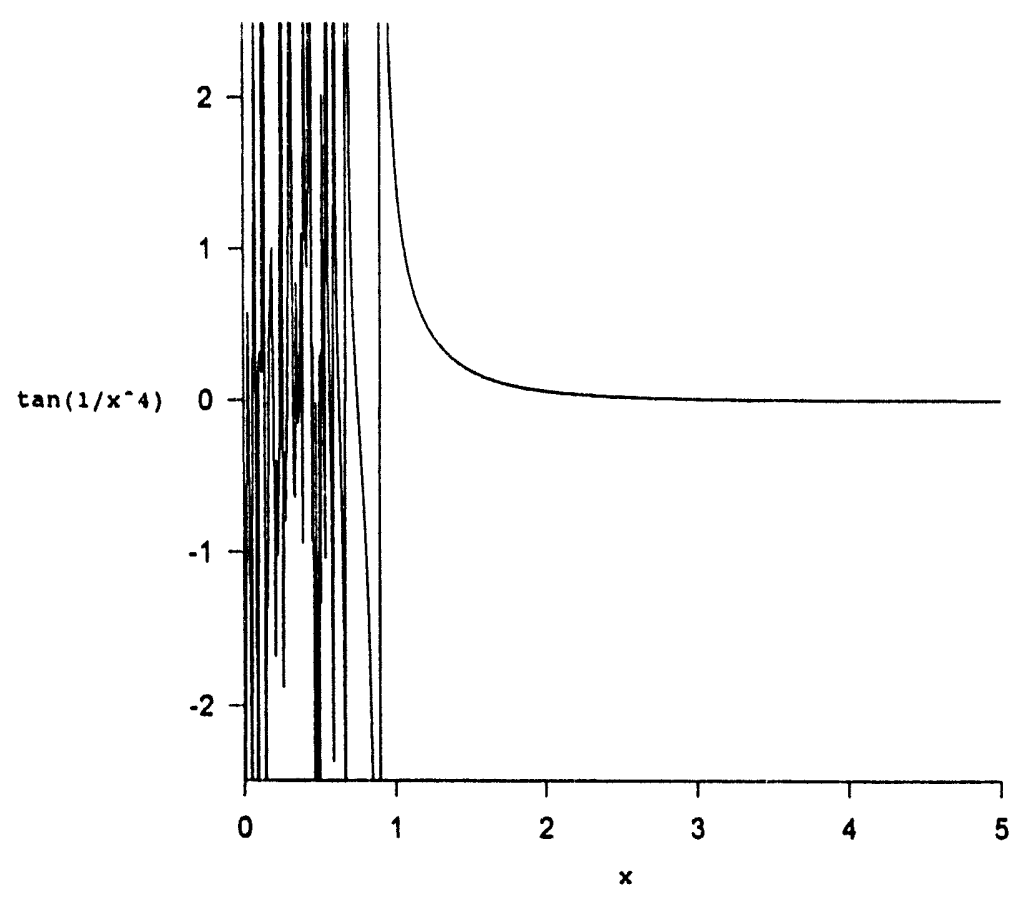

Figure 16. Plot of $\tan \left(1 / x^{4}\right)$ for $0<x<5$

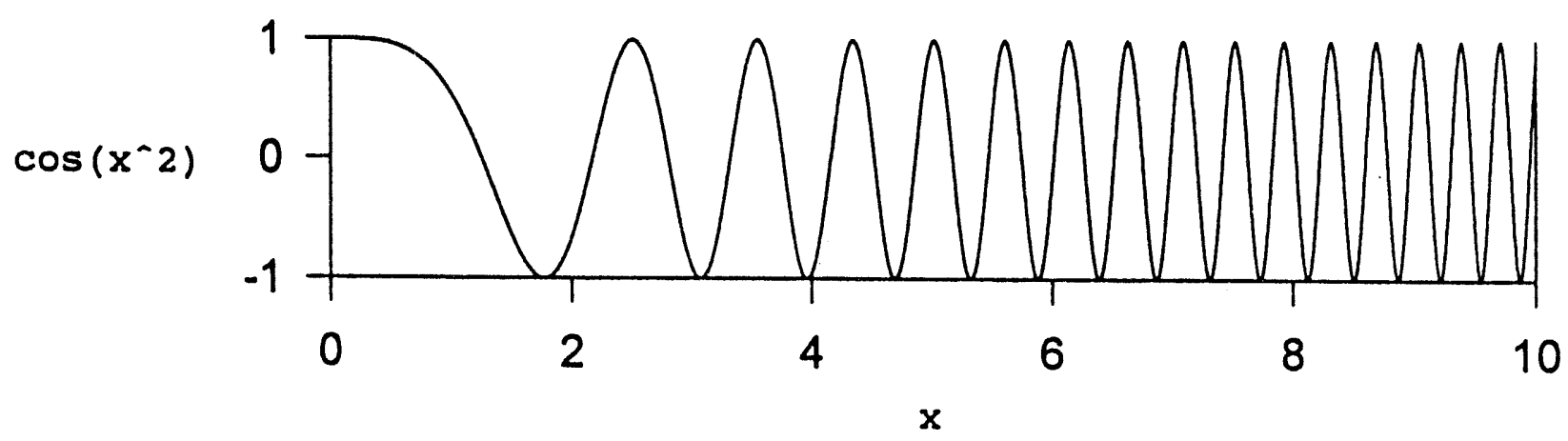

Figure 17. Plot of $\cos \left(\mathrm{x}^{2}\right)$ for $0<\mathrm{x}<10$ 


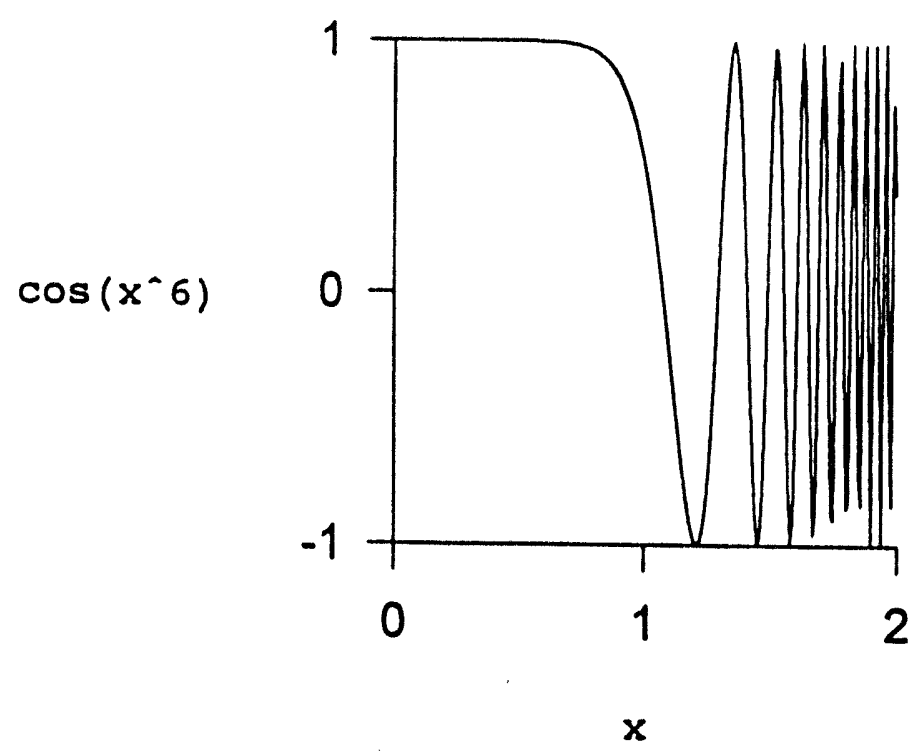

Figure 18. Plot of $\cos \left(x^{6}\right)$ for $0<x<2$

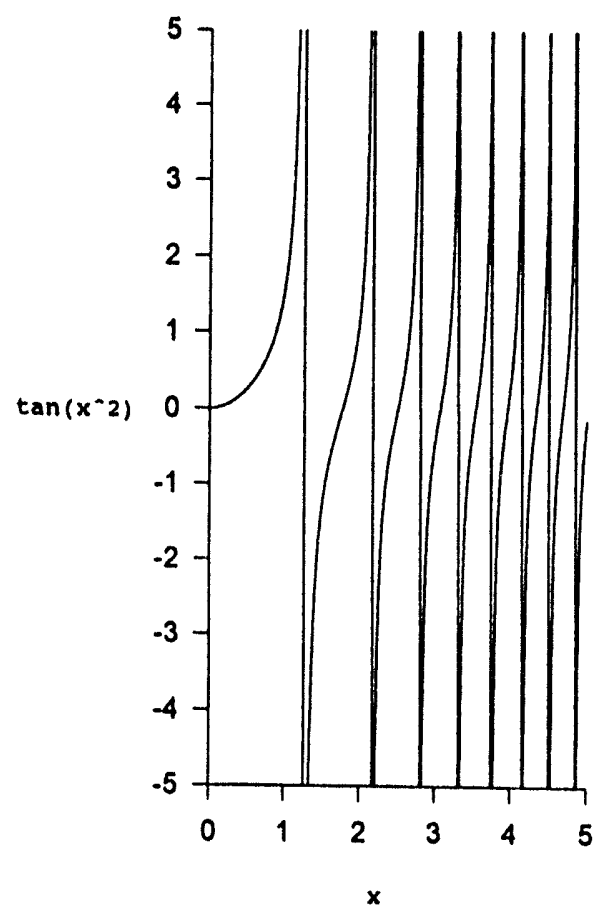

Figure 19. Plot of $\tan \left(x^{2}\right)$ for $0<x<5$ 


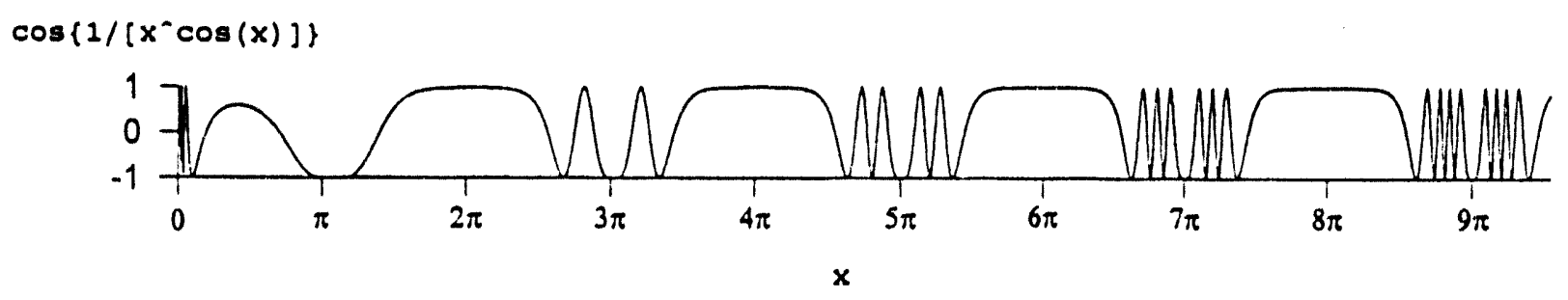

Figure 20. Plot of $\cos \left\{1 /\left[x^{\cos (x)}\right]\right\}$

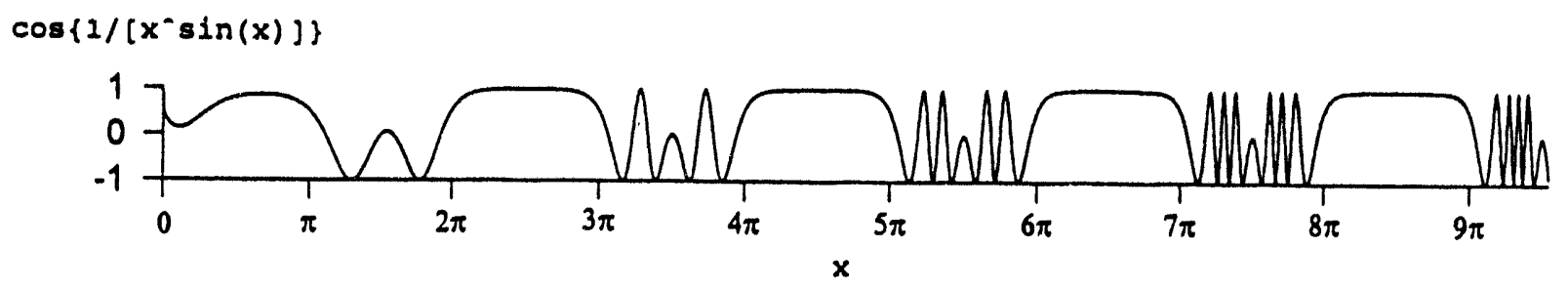

Figure 21. Plot of $\cos \left\{1 /\left[\mathrm{x}^{\sin (x)}\right]\right\}$

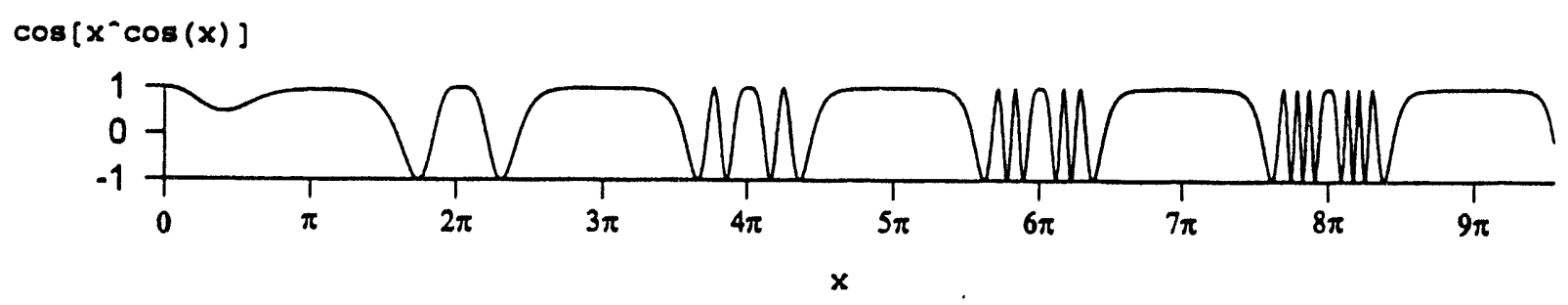

Figure 22. Plot of $\cos \left[x^{\cos (x)}\right]$

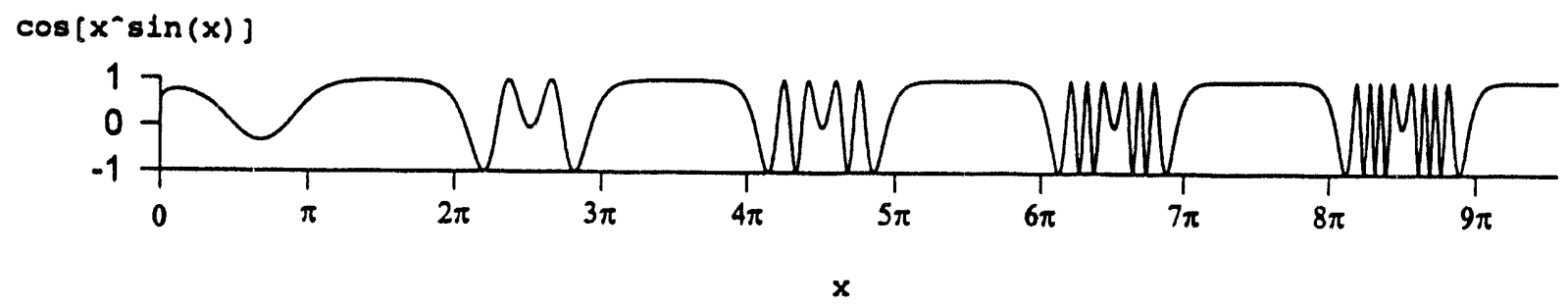

Figure 23. Plot of $\cos \left[x^{\sin (x)}\right]$ 


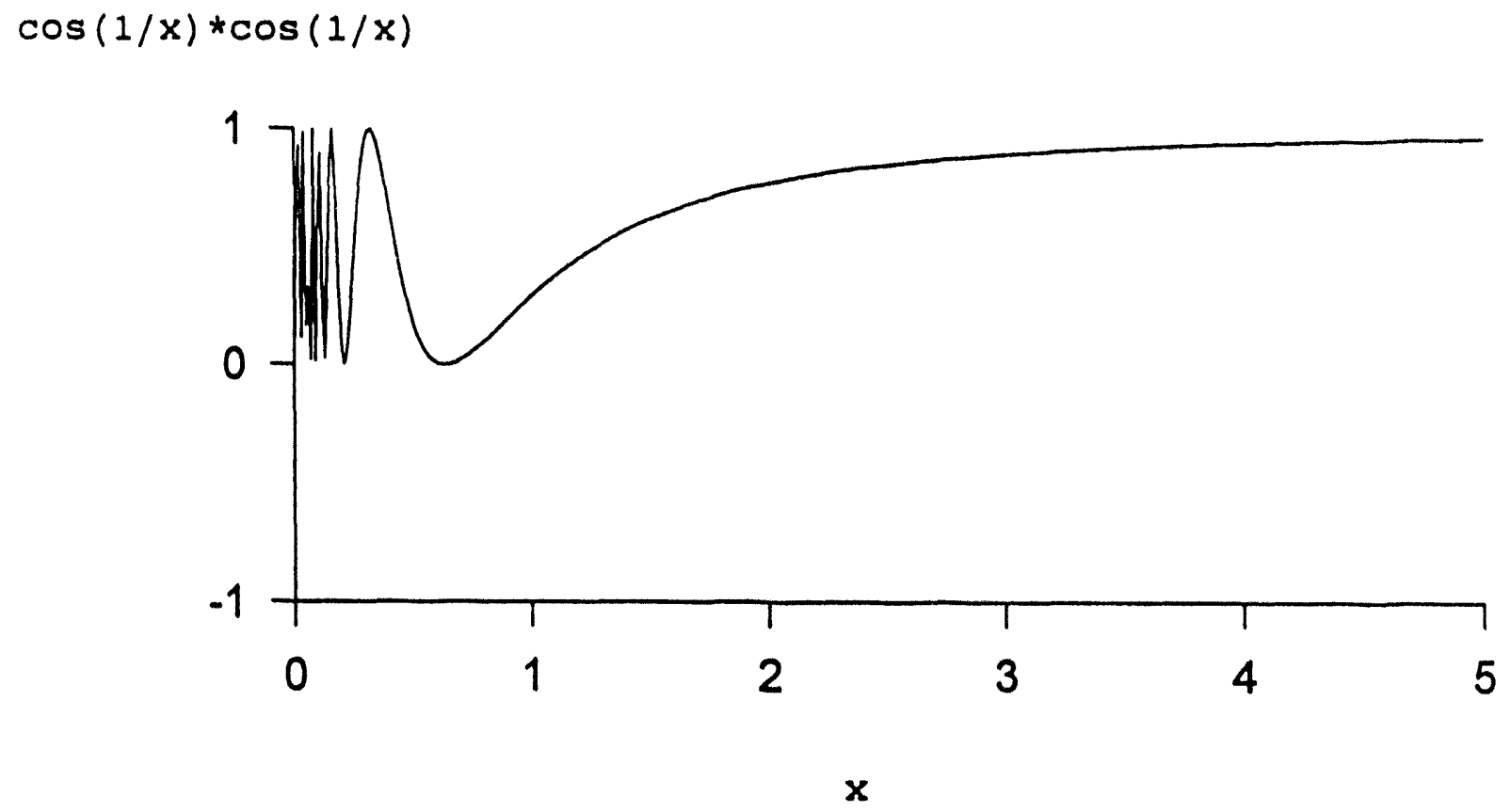

Figure 24. Plot of $\cos (1 / x) \cdot \cos (1 / x)$ for $0<x<5$

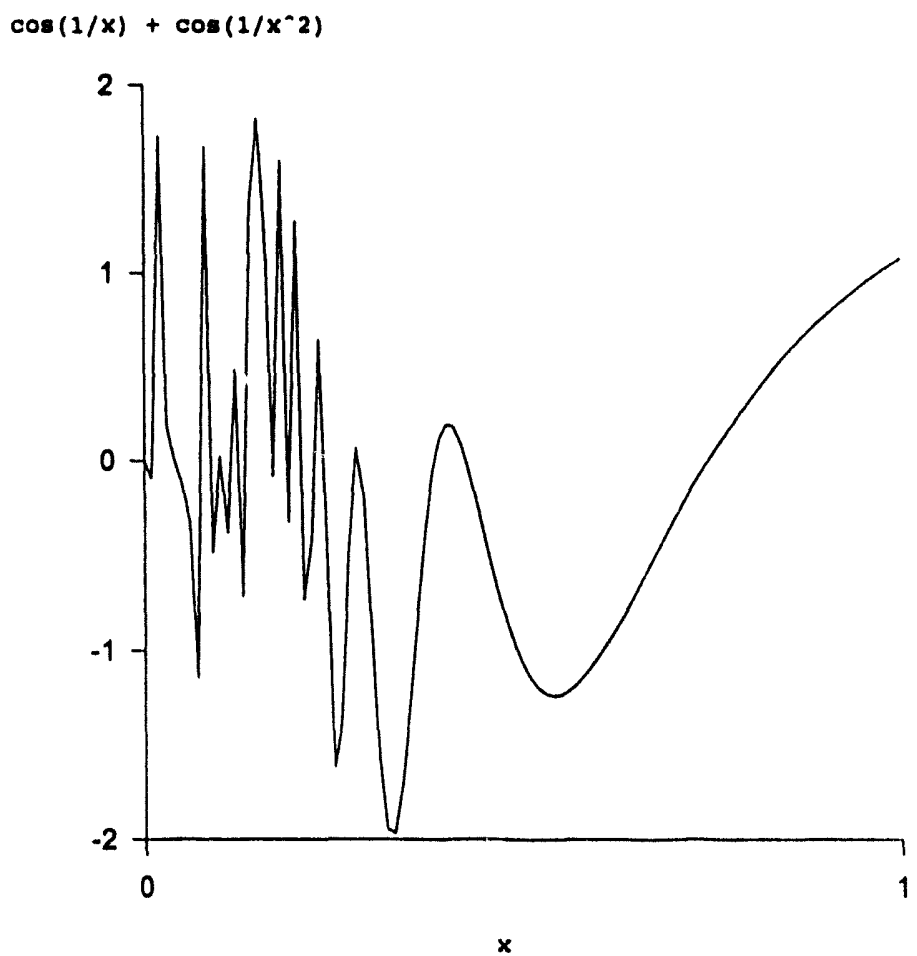

Figure 25. Plot of $\cos (1 / x)+\cos \left(1 / x^{2}\right)$ for $0<x<1($ expanded $x)$ 


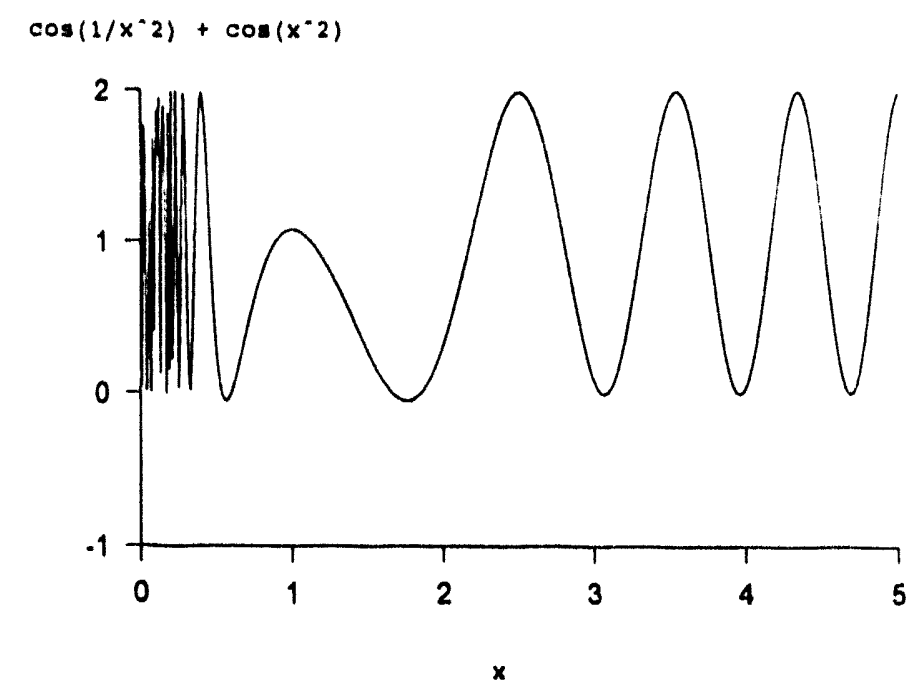

Figure 26. Plot of $\cos \left(1 / \mathrm{x}^{2}\right)+\cos \left(\mathrm{x}^{2}\right)$ for $0<\mathrm{x}<5$

$\cos (1 / x) \cdot \cos \left(1 / x^{-} 2\right)$

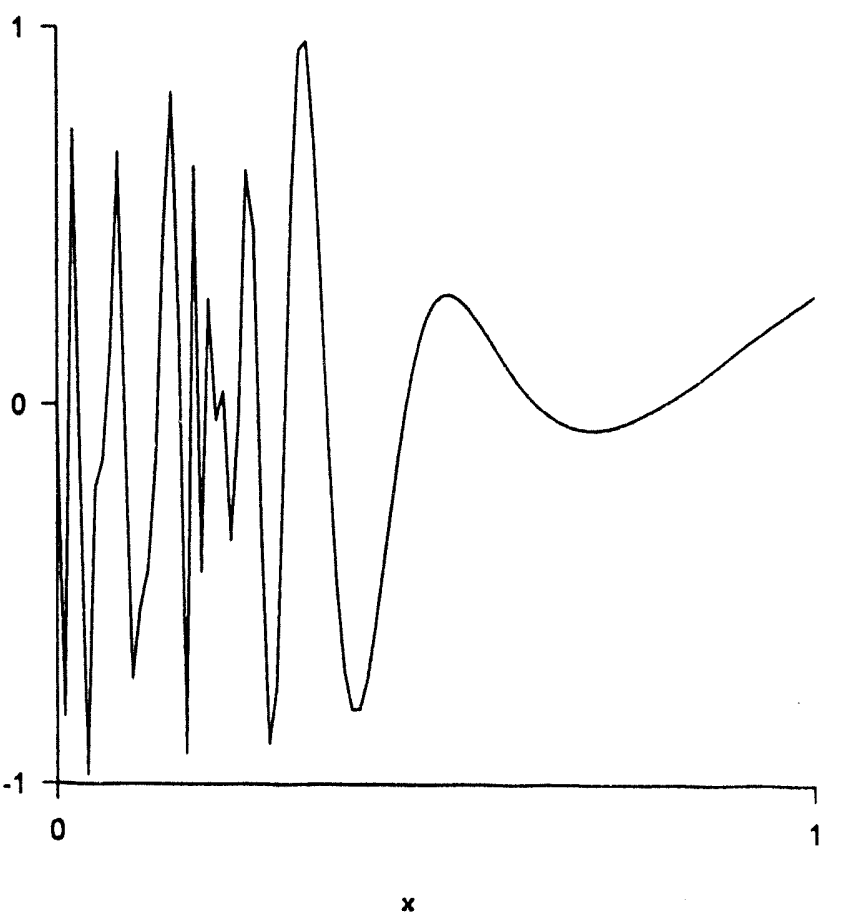

Figure 27. Plot of $\cos (1 / x) \cdot \cos \left(1 / x^{2}\right)$ for $0<x<1($ expanded $x)$ 


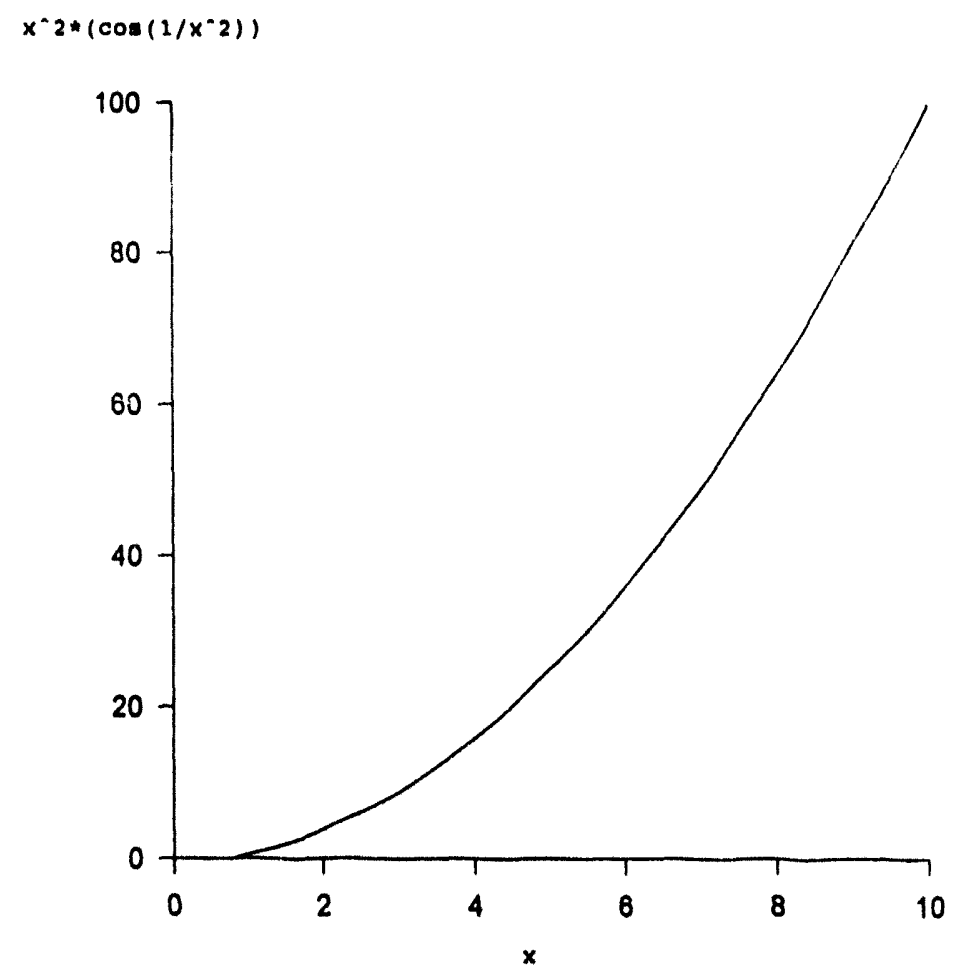

Figure 28. Plot of $x^{2} \cdot\left(\cos \left(1 / x^{2}\right)\right)$ for $0<x<10$

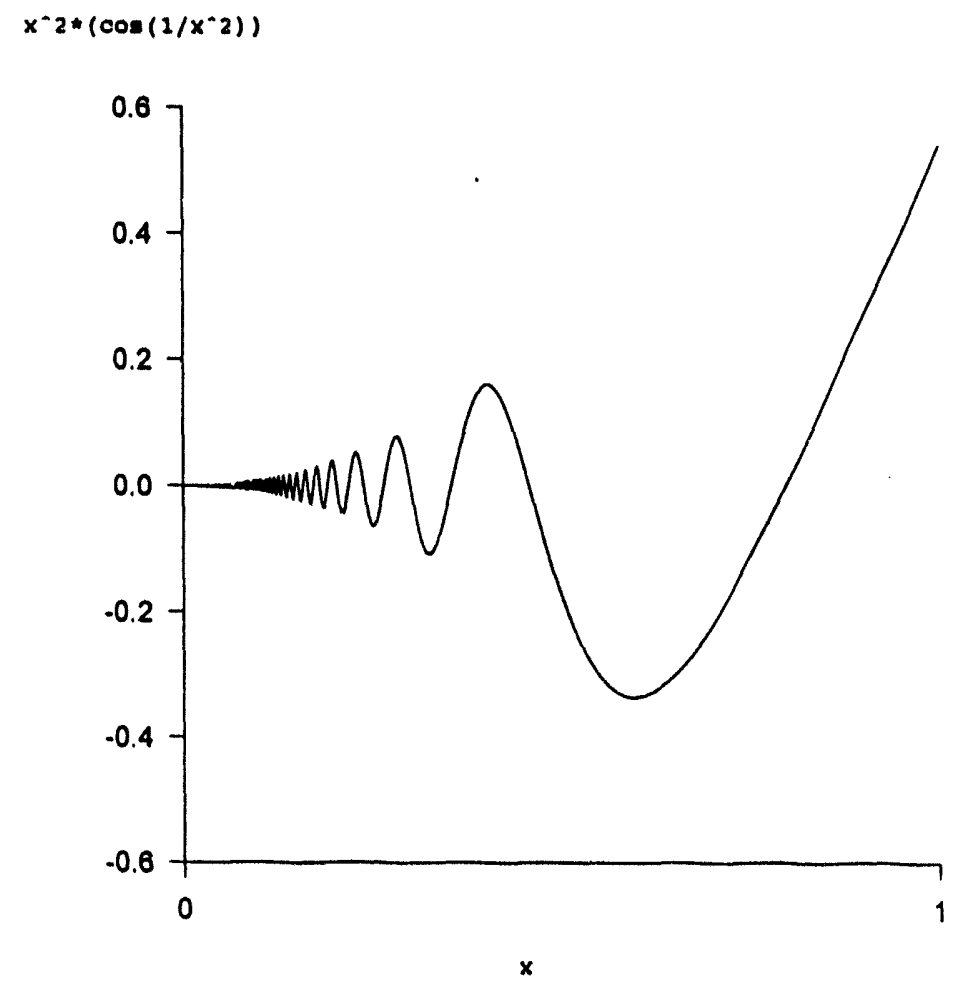

Figure 29. Plot of $x^{2} \cdot\left(\cos \left(1 / x^{2}\right)\right)$ for $0<x<1$ 


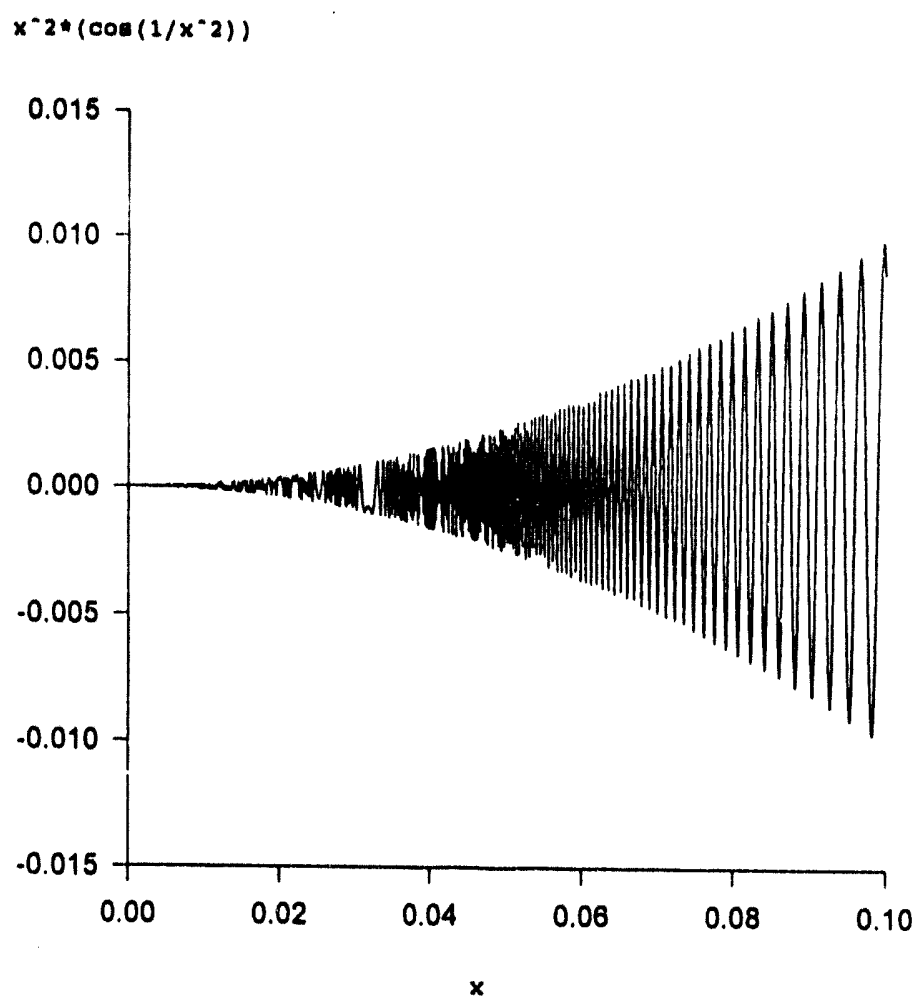

Figure 30. Plot of $x^{2} \cdot\left(\cos \left(1 / x^{2}\right)\right)$ for $0<x<0.1$ 


\section{Distribution}

No. of

Copies

Offsite

2 DOE/Office of Scientific and Technical Information

\section{Onsite}

32 Pacific Northwest Laboratory

G. H. Bryan, P7-25

B. C. Bunker, K2-45

L. L. Burger, P7-25

H. D. Collins, K2-31

T. H. Dunning, K2-20
No. of

Copies

E. A. Eschbach, K1-24

B. C. Garrett, K1-90

M. L. Knotek, K1-48

W. W. Laity, K2-50

R. J. Littlefield, K1-87

G. J. Lumetta, P7-25

D. L. McMakin, K2-31

A. C. Rither, K1-67

G. K. Schenter, K1-90

L. J. Sealock, K2-10

D. W. Wester, P7-25 (10)

P. W. Zimmerman, K1-53

Publishing Coordination

Technical Report Files (5) 

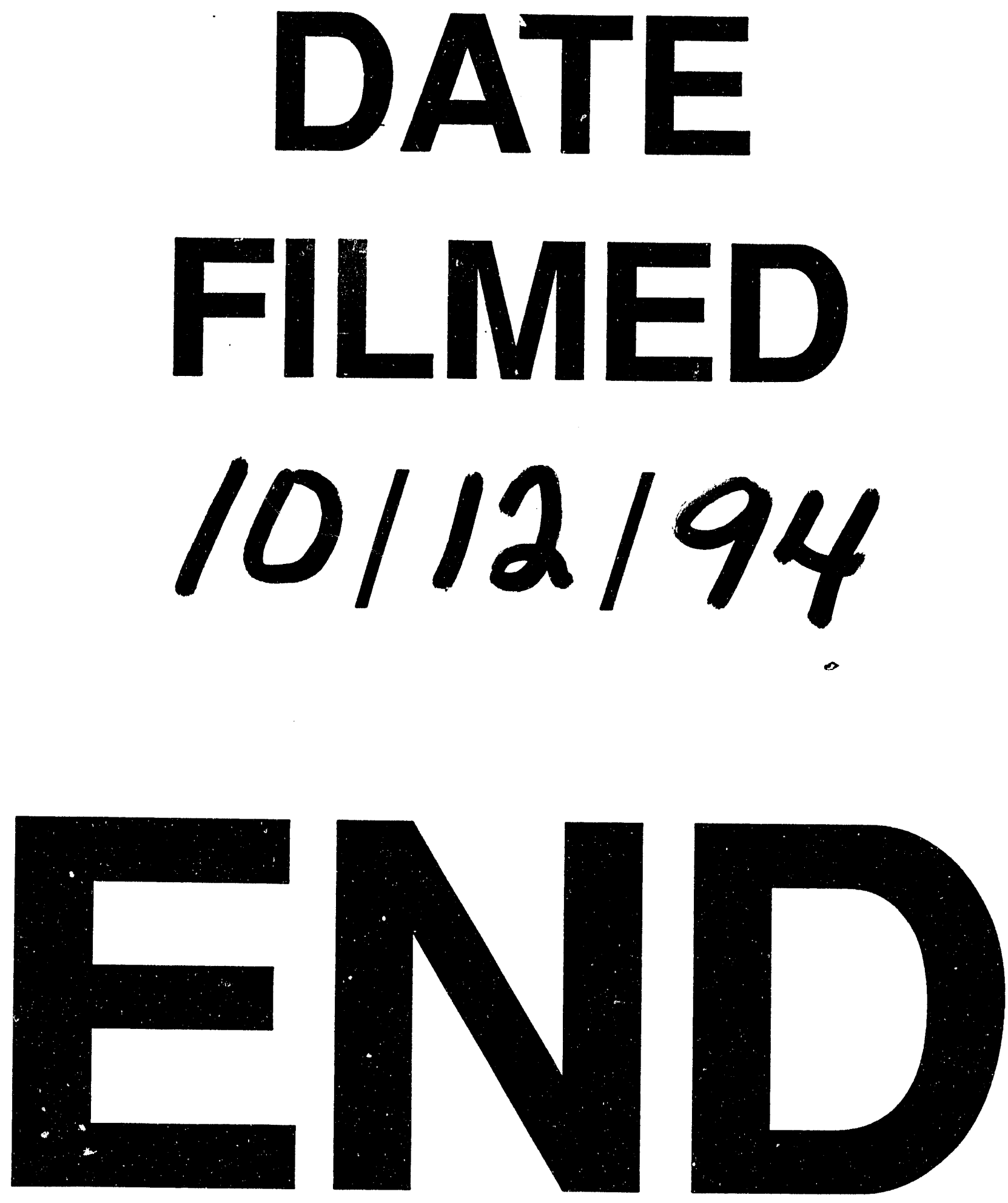
